\title{
QED challenges at FCC-ee precision measurements
}

\author{
S. Jadach ${ }^{\mathrm{a}} \mathbb{1}$, M. Skrzypek \\ Institute of Nuclear Physics, Polish Academy of Sciences, ul. Radzikowskiego 152, 31-342 Kraków, Poland
}

Received: 6 June 2019 / Accepted: 27 August 2019 / Published online: 11 September 2019

(c) The Author(s) 2019

\begin{abstract}
The expected experimental precision of the rates and asymmetries in the Future Circular Collider with electron-positron beams (FCC-ee) in the center of the mass energy range 88-365 GeV considered for construction in CERN, will be better by a factor 5-200. This will be thanks to the very high luminosity, a factor up to $10^{5}$ higher than in the past LEP experiments. Consequently, it poses the extraordinary challenge of improving the precision of the Standard Model predictions by a comparable factor. In particular the perturbative calculations of the trivial QED effects, which have to be removed from the experimental data, are considered to be a major challenge for almost all quantities to be measured at FCC-ee. The task of this paper is to summarize the "state of the art" in this class of the calculations left over from the LEP era and to examine what is to be done to match the precision of the FCC-ee experiments - what kind of technical advancements are necessary. The above analysis will be done for most important observables of the FCC-ee, like the total cross sections near $Z$ and $W W$ threshold, charge asymmetries, the invisible width of $Z$ boson, the spin asymmetry from $\tau$ lepton decay and the luminosity measurement.
\end{abstract}

\section{Contents}

1 Introduction . . . . . . . . . . . . . 1

2 General features of QED corrections . . . . . . . 2

2.1 Optimal strategy for advancing QED precision . 3

2.2 More on soft and collinear resummation in QED calculations ............ 4

2.3 QED "deconvolution" . . . . . . . . . . . . 5

3 The most important QED-sensitive experimental observables at LEP and FCC-ee . . . . . . . . . . 6

This work is partly supported by the Polish National Science Center Grant 2016/23/B/ST2/03927 and the CERN FCC Design Study Programme.

a e-mail: stanislaw.jadach@ifj.edu.pl
4 Present state of the art in calculating QED effects . . 7

4.1 Cross section near the $Z$ resonance: mass and width of $Z \ldots \ldots \ldots \ldots \ldots$. . . . . . 7

4.2 Luminosity measurement . . . . . . . . . . 8

4.3 EW mixing angle from charge and spin asymmetries at LEP . . . . . . . . . . . . . . 8

4.4 The invisible $Z$ decay width from cross section and radiative return in LEP experiments . . . . 9

4.5 QED in $W$-pair production at LEP, $M_{W}$ measurement 10

5 Prospects of the QED calculation improvements in the FCC-ee measurements . . . . . . . . . . . . 12

5.1 Cross section near the $Z$ resonance and mass of $Z 12$

5.2 Charge and spin asymmetries at FCC-ee . . . . 13

5.3 Luminosity measurement . . . . . . . . . . . 15

5.4 Measurement of $\alpha_{Q E D}\left(M_{Z}\right)$ using $A_{\mathrm{FB}}$ near $Z$ resonance ............ 16

5.5 Invisible $Z$ decay width from cross section and radiative return . . . . . . . . . . 16

5.6 Cross section near the $W W$ threshold ( $M_{W}$ measurement) . . . . . . . . . . . . 17

5.7 New ideas on pseudo-observables at FCC-ee . . 19

6 Summary . . . . . . . . . . . . . . . . . 20 20

References . . . . . . . . . . . . . . . 20

\section{Introduction}

The high-energy high-luminosity future circular electronpositron collider FCC-ee [1-3], considered for construction at CERN, would feature a luminosity up to a factor $10^{5}$ higher than at the LEP collider. Together with the improvements of the detector techniques it would allow one to reduce an experimental error typically by a factor of 5-50, and in some cases even by a factor of 200 . The uncertainty of the corrections due to trivial but large QED effects to be removed from the data would then become for many observables a dominant one and its reduction becomes a major challenge at the FCC-ee programme. For instance, some QED effects of order $0.1 \%$, 
which at LEP could be neglected and accounted for in the error budget, will have to be calculated with two-digit precision, removed from the data and their uncertainty hopefully below $0.001 \%$ will enter into a combined experimental and theoretical error. It is therefore important to review already now the present state of the art in the precise calculations of the SM for $e^{+} e^{-}$annihilation processes left over from the LEP era not only for multiloop pure electroweak corrections [4-6], but also for QED effects and to evaluate the prospects of the necessary future improvements in this area of the theoretical physics.

The modern techniques of calculating QED corrections in electron-positron colliders for arbitrary experimental cutoffs using Monte Carlo event generators were founded in the 1980s for use in the PETRA and PEP experiments. Typically, these calculations were implementing complete $\mathcal{O}\left(\alpha^{1}\right)$. Their precision tag (neglected higher orders) was of order 1-2\%; see for instance Ref. [7]. Analytic phase space integration of QED distributions was not playing at that time any major role, apart from being used in designing Monte Carlo algorithms. The LEP era has seen development of the entire new range of QED calculations for many new processes, mostly in the form of the MC event generators. However, for the analyzing of data near the $Z$ resonance, numerical programs based on analytic integration over the phase space were also playing an important role. Also first QED calculations at the $\mathcal{O}\left(\alpha^{2}\right)$ have appeared [8], but the biggest boost in the precision came from resummation to infinite order of soft photon corrections [9] and from the calculations of the collinear logs due to small lepton masses to higher orders, up to $\mathcal{O}\left(\alpha^{3}\right)$ [10]. Several of the MC programs were also armed with the complete $\mathcal{O}\left(\alpha^{1}\right)$ electroweak (EW) corrections. The example of the most sophisticated MC event generator from the LEP era with soft photon resummation, complete $\mathcal{O}\left(\alpha^{2}\right)$ QED and $\mathcal{O}\left(\alpha^{1}\right)$ EW corrections is KKMC program [11]. Generally, in the LEP data analysis near the $Z$ peak, the use of MC generators was often limited to removing detector inefficiencies and partly removing experimental cut-off effects. Fitting EW parameters like the masses of the top quark and the Higgs boson was done with non-MC programs like ZFITTER [12] and TOPAZ 0 [13]. However, at the LEP2 above the $W W$ threshold, $M C$ event generators $[14,15]$ were the only tools capable to calculate QED+EW Standard Model predictions for the total cross section and distributions of the $e^{+} e^{-} \rightarrow W^{+} W^{-}$ process and also were used to extract (fit) the mass of the $W$ boson from the data.

As it will be argued in the following, due to significant increase of the experimental and statistical precision at FCC-ee, the role of the MC event generators will increase. Near $Z$ resonance a combination of $\mathrm{MC}$ programs and semianalytical non-MC calculations will be used in the data anal- ysis [4]. ${ }^{1}$ This will be due to many reasons: the increase of non-factorizable QED corrections above the level of the experimental precision, the need of further development of the resummation techniques of soft and collinear corrections which will be the only available within MC programs and the generally bigger role of the multiparticle final states, for which the MC technique is the only method of integration over the phase space. The future tools and techniques for practical calculations of the QED effects will gradually emerge in more detail in the following discussion of many observables to be measured at FCC-ee.

The content of the paper is the following: we start with a brief overview of perturbative QED techniques. In particular the soft and collinear factorization is substantially different from that of QCD. It will be stressed that an optimal strategy of truncation of the perturbative orders from the point of view of precision is not the simplistic order-by-order truncation but a more subtle approach taking into account mass logarithms at higher orders. Next we shall elaborate on the QED component of the systematic errors in the measurements of the mass and partial width of $Z$, the total cross section and asymmetries near the $Z$ resonance and of the $W$ mass measurement, according to the present state of art inherited from the LEP era, describing in detail the main source of QED uncertainties in these experimental observables. Next, we shall compare present QED precision of the observables with the planned experimental systematic and statistical precision at FCC-ee, in order to estimate how much improvement is needed in the QED calculations. For each experimental observable we shall examine how difficult it will be to get sufficient improvement in terms of more perturbative orders, improvement in the resummation techniques and development of new software tools i.e. the entire new class of dedicated MC event generators. In the above discussion we shall briefly elaborate upon the question of how to factorize in practice the resummed QED and the so-called "pure electroweak" parts of the perturbative calculation such that they coexist in the $\mathrm{MC}$ event generators forming a complete perturbative expansion without any double counting nor under counting. A short summary will complete the paper.

\section{General features of QED corrections}

Figure 1 shows parameters which control effectively the strength of the QED $\mathcal{O}\left(\alpha^{n}\right)$ corrections for $e^{ \pm}$beams (ISR) and for final state muon pair $\mu^{ \pm}$(FSR) at the $Z$ peak:

$\gamma_{n r}=\left(\frac{\alpha}{\pi}\right)^{n}\left(2 \ln \frac{M_{Z}^{2}}{m_{f}^{2}}\right)^{r}, \quad 0 \leq r \leq n$,

\footnotetext{
1 The most important role of semi-analytical calculations will be in testing/validating MC programs.
} 
Fig. 1 The parameter $\gamma_{n r}$ of Eq. (2.1) characterizing the size of the QED corrections

Fig. 2 QED perturbative leading and subleading corrections. Rows represent corrections in consecutive perturbative orders - the first row is the Born contribution. The first column represents the leading logarithmic (LO) approximation and the second column depicts the next-to-leading (NLO) approximation. In the figure, terms selected for the same precision level are limited with the help of an additional line
QED strength, ISR $\mathrm{e}^{+} \mathrm{e}^{-}$

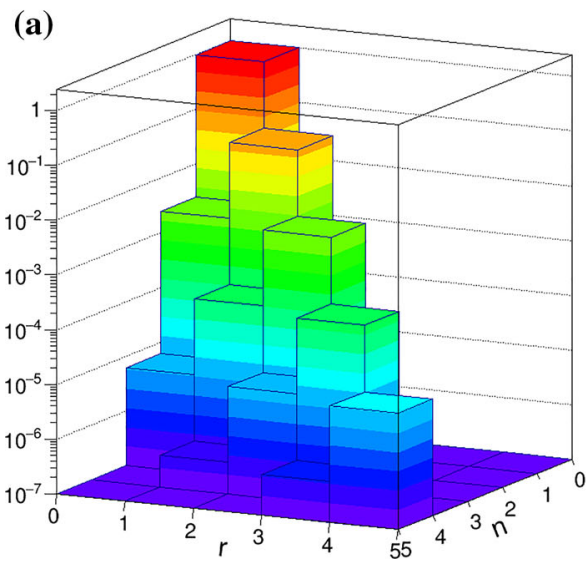

(a) $0.6 \%$

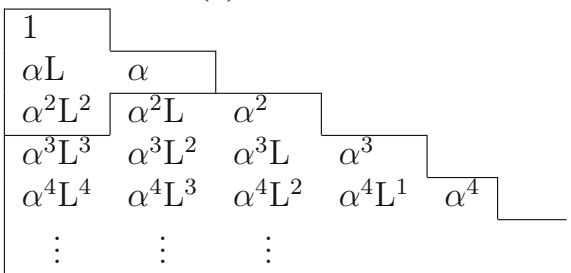

(c) $0.001 \%$
QED strength, FSR $\mu^{+} \mu^{-}$

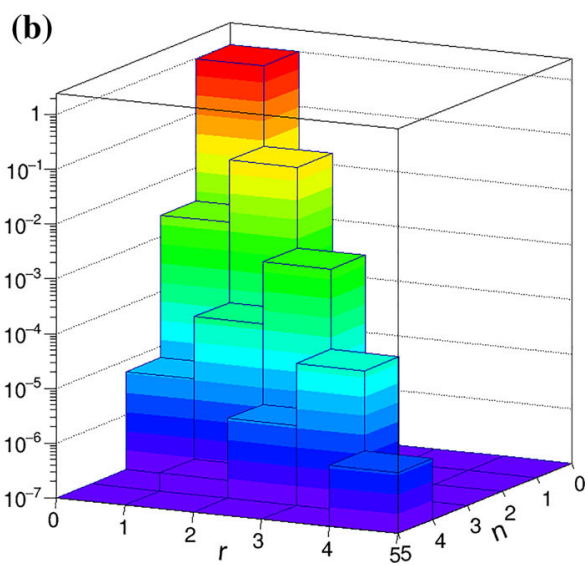

(b) $0.03 \%$

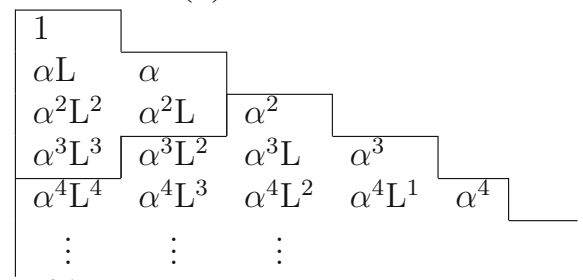

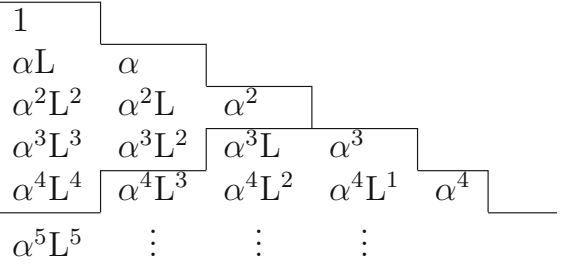

for $f=e, \mu$, respectively. The relative precision $6 \cdot 10^{-3}$ requires the inclusion of corrections of the QED correction up to

$\mathcal{O}\left(\alpha^{1} L_{f}^{1}, \alpha^{1} L_{f}^{0}, \alpha^{2} L_{f}^{2}\right), \quad L_{f}=\ln \left(s / m_{f}^{2}\right)$,

while the remaining second order correction $\mathcal{O}\left(\alpha^{2} L_{f}^{1}, \alpha^{2} L_{f}^{0}\right)$ can be safely neglected. This is also visualized in Fig. 2a.

The next precision level of $3 \cdot 10^{-4}$ requires adding $\mathcal{O}\left(\alpha^{3} L_{f}^{3}, \alpha^{2} L_{f}^{1}\right)$; see also Fig. $2 \mathrm{~b}$ for the illustration. It is only at the precision level $1 \cdot 10^{-5}$ (relevant for the FCC-ee) that including $\mathcal{O}\left(\alpha^{2} L_{f}^{0}\right)$ looks necessary, but it should to be accompanied in addition with $\mathcal{O}\left(\alpha^{4} L_{f}^{4}, \alpha^{3} L_{f}^{2}\right)$ corrections in order to be useful in practice.

The case of muon pair differs only slightly, as can be read from the RHS plot in Fig. 1.

\subsection{Optimal strategy for advancing QED precision}

In view of the above estimates of the numerical importance of the generic perturbative QED contributions it is easy to answer the question of the optimal strategy for advancing the precision of QED calculations. For example, performing

the complete $\mathcal{O}\left(\alpha^{3}\right)$ calculation may look justified because it will be automatically gauge invariant, all soft/collinear limits and mass effects will be correct. However, for a precision at the $\sim 0.5 \%$ level, a much simpler (almost trivial) calculation taking only up to $\mathcal{O}\left(\alpha^{2} L_{f}^{2}\right)$ into account is enough; see Fig. 1 . Similarly, the inclusion of $\mathcal{O}\left(\alpha^{2} L_{f}^{1}\right)$ in order to gain precision $\sim 0.05 \%$ level is in practice useless, unless an $\mathcal{O}\left(\alpha^{3} L_{f}^{3}\right)$ contribution enters into the game; see Fig. 1.

It is also a common misconception that including $\mathcal{O}\left(\alpha^{2} L_{f}^{2}\right)$ forces us into the use of the strictly collinear approximation. ${ }^{2}$ It was demonstrated that in the EEX matrix element [16] with $\mathcal{O}\left(\alpha^{2} L_{f}^{2}, \alpha^{3} L_{f}^{3}\right)$ corrections can be implemented at the exclusive (unintegrated) level, i.e. keeping angle distributions for two and more photons accessible for experimental cut-offs.

\footnotetext{
2 By "strictly collinear" we mean that in the structure function photon angle is integrated over, i.e. photon is treated partly inclusively.
} 
2.2 More on soft and collinear resummation in QED calculations

The other important question is that of the importance of the soft and collinear photon resummation. For totally inclusive observables in the final state (like FSR in the $Z$ boson decay) mass logarithms do cancel completely among real and virtual ones according to the Kinoshita-Lee-Nauenberg theorem. For the cut-off on the total photon energy $E_{\max } \ll E_{\text {beam }}$, the $\mathcal{O}\left(\alpha^{n}\right)$ YFS/Sudakov double logarithmic contributions

$S^{(n)} \sim\left(\frac{\alpha}{\pi} 2 L_{f} \ln \frac{E_{\text {max }}}{E_{\text {beam }}}\right)^{n}$

for a strong cut-off may easily be huge, $S^{(n)} \gg 1$, and they definitely require resummation. Only for a loose cut-off like $E_{\text {max }} / E_{\text {beam }} \sim 0.2$ and very low precision requirements one could avoid soft photon resummation.

There are several techniques of soft photon resummation of the different levels of sophistication. In the most primitive version all photon energies are restricted to small values, the integration over photon angles and energies is done analytically, keeping only the sum of photon energies fixed. This we call inclusive soft photon exponentiation, IEX in short. In the work of Yennie-Frautschi-Suura (YFS) [17] it was outlined how to match smoothly soft and hard photon distributions, covering the entire phase space, without any need of the cutoff on total photon energy. It was only in the late 1980s that it was gradually worked out how to implement YFS soft photon factorization and resummation within the Monte Carlo event generators $[18,19]$. This technique we call exclusive photon exponentiation, EEX in short. In the EEX methodology soft photon factorization and resummation is done at the level of the multiphoton fully differential distributions, that is, for spin amplitudes squared and spin summed/averaged.

Near the narrow resonances $R$, a similar class of soft logarithms,

$S^{(n)} \sim\left(\frac{\alpha}{\pi} \ln \frac{\Gamma_{R}}{M_{R}}\right)^{n}$,

present in real and virtual corrections also requires resummation. In addition, due to the complicated pattern of the QED interferences between the initial and final state photons (cancellations due to short lifetime of the resonance) which operate at the amplitude level, one is forced to perform soft photon factorization at the amplitude level, before squaring and spin summing. This technique was developed by the Frascati group [20]. Moreover, the need of proper implementation of spin effects in the $\tau$ pair production and decays at LEP also was enforcing the use of spin amplitudes. In order to meet the above requirements a new variant of YFS-inspired soft photon resummation was developed [21], in which soft photon factorization was reformulated at the amplitude level and soft photon resummation is implemented numerically within the MC program. Narrow resonance effects were accommodated as in Refs. [20,22]. This technique we call coherent exclusive exponentiation, CEEX in short. So far, the only implementation of CEEX technique is in the KKMC event generator [11].

The important message to theorists specialising in QED+EW multiloop calculations is the following: do not add soft real emissions to multiloop results in order to eliminate infrared singularities á la Bloch-Nordsieck, if you want these results to be used in the MC generators with IR resummation. Instead, you should subtract IR parts (YFS virtual formfactor) from the amplitudes, before squaring and spin summing. ${ }^{3}$ Why? Because combining IR soft and real contributions and the differential cross-section level is already done in the Monte Carlo.

The related important practical question is whether the use of collinear resummation of the mass logarithms is mandatory in QED to infinite order, like in QCD? Obviously in QED it is not mandatory and in practice it is usually enough to stop at some finite order, typically truncating infinite LO series at $\mathcal{O}\left(\alpha^{3} L_{f}^{3}\right)$. In the FCC-ee environment it may be sometimes necessary to include $\mathcal{O}\left(\alpha^{4} L_{f}^{4}\right)$.

In the case of the photon emission from leptons, employing the entire machinery of the collinear resummation technique up to $\mathrm{LO}+\mathrm{NLO}$ or $\mathrm{LO}+\mathrm{NLO}+\mathrm{NNLO}$ level is not trivial due to finite lepton masses, handling properly the factorization scale parameter, sectorization of the phase space etc. In practice, it is more convenient and/or economical to perform soft photon resummation in the first place and only then to include collinear resummation truncated to a convenient order. ${ }^{4}$ This technique was used successfully in the many MC projects like KORALZ [23], KKMC [11], BHLUMI [24], YFSWW [25], KORALW [26], BHWIDE [27], YFSZZ [28], also in the semi-analytic approaches like ZFITTER [12] and TOPAZO [13]. Moreover, soft photon resummation is of the highest possible priority for the resonant processes, obviously near the $Z$ resonance, but also it will be mandatory in the $W W$ production process, especially near the $W W$ threshold.

Summarizing the above discussion:

- The (complete) order-by-order perturbative calculation in QED is definitely not the economic way to obtain pre-

\footnotetext{
3 One can do it for gauge invariant groups of diagrams but not for individual diagrams. Undoing Bloch-Nordsieck at $\mathcal{O}\left(\alpha^{2}\right)$ is usually unfeasible, rendering two-loop calculations useless for the MC.

4 Due to the more complicated infrared limit and almost zero quark masses, resummation in QCD is done in different order - collinear resummation and soft resummation are in principle done simultaneously, with soft limit approximated quite often to some convenient order within the leading logarithmic expansion.
} 
dictions for cross sections or asymmetries with the precision below $0.1 \%$.

- Soft photon resummation is an absolute necessity, especially for resonant processes. It exists in at least three different variants (IEX, EEX and CEEX).

- Do not follow Bloch-Nordsieck to eliminate IR singularities!

- Resummation of collinear mass $\operatorname{logarithms} \ln \left(s / m_{f}^{2}\right)$ is very useful, but in QED it is usually convenient to truncate it at some finite order.

- Approximation of small lepton mass $m_{f}^{2} / s \ll 1$ should be exploited for electron and muons as much as possible, but for $\tau$ lepton $\propto m_{\tau}^{2} / s, m_{\tau}^{4} / s^{2}$ terms may not be negligible at tree level, while higher powers of this type in higher orders of $\alpha$ are probably irrelevant.

\subsection{QED “deconvolution”}

The so-called "deconvolution" of QED effects is the procedure of removing universal (process independent) QED effects from experimental data for the total cross section and angular differential cross sections in the process $e^{+} e^{-} \rightarrow$ $f \bar{f}(+n \gamma)$. It was a cornerstone of the final analysis of LEP data near the $Z$ peak in Ref. [29]. Cross sections, asymmetries, branching ratios, $Z$ mass and partial widths derived from the "deconvoluted" integrated/differential experimental distributions were called EW pseudo-observables (EWPOs) and were (approximately) independent of the QED effects and experimental cut-offs, such that they could be combined among four LEP collaboration and SLD. The technique of EWPOs was defined and thoroughly elaborated in Ref. [30].

N.B. The term "deconvolution" is a little bit misleading, because in practice it means fitting a certain theoretical formula to experimental data at one or several energies. ${ }^{5}$

It is tempting to assume that at FCC-ee one can use the same QED deconvolution as at LEP. However, this assumption may be wrong due to the much higher experimental precision at FCC-ee.

Let us comment briefly on some aspects of the factorization of the soft and collinear QED corrections, which is the basis of QED deconvolution and an essential element in the construction of EWPOs. This is because one could worry that the entanglement of electromagnetic and weak interactions at multiloop corrections, the rise of non-factorizable interferences above the level of FCC-ee precision, may give rise to practical or principal problems with the clean separation of the QED universal corrections from the complete EW perturbative calculations, especially beyond the 1-st order.

\footnotetext{
5 The true deconvolution would require taking data for $d \sigma(s) / d \cos \theta$ on a dense grid of $s$ and $\theta$ and calculating the deconvoluted differential distribution at $s$ by means of combining all data $s^{\prime}<s$, using some weight provided by QED, without any fitting.
}

In Refs. [29,30] the simple version of QED ISR deconvolution relies on a well known simple convolution formula with the integration over a single variable (total ISR photon energy) over the product of two objects: the ISR radiator function $^{6}$ and Born-like differential or integrated cross section. In the case of a cut-off on the final fermion pair effective mass, the integration over the radiator function of FSR had to be included. The resulting double convolution formula for ISR $\times$ FSR was used in Ref. [30] and also in Ref. [16] for testing KKMC. In addition, in the ZFITTER and TOPAZO programs missing $\mathcal{O}\left(\alpha^{1}\right)$ contributions were added into the game. In particular, missing initial-final state interference (IFI) and effects due to experimental cut-offs were also added in this way.

As discussed in Ref. [30], the above simple treatment of IFI at LEP analysis was possible because near the $Z$ peak, in the absence of strong cut-offs IFI is suppressed by an additional $\Gamma_{Z} / M_{Z}$ factor, while away from the $Z$ peak, where LEP data were of limited precision and IFI also could be either neglected or eliminated using an additive $\mathcal{O}\left(\alpha^{1}\right)$ correction. The above treatment of IFI would be highly unsatisfactory at the FCC-ee precision.

The first mandatory thing on the way to an improved treatment of IFI is soft photon resummation. The multiphoton convolution formula at the level of the matrix element in the soft photon approximation was constructed a long time before the LEP era, by the Frascati group [20,32], and it is a natural extension of the exponentiation formula of YennieFrautschi-Suura [17] to a resonant process.

In the Frascati-type formula with soft photon resummation at the amplitude level the effective Born matrix element is clearly factorized out and could be exploited for constructing a better variant of the EWPO definition. However, as was shown in Ref. [33], after squaring, spin summing, and integrating over photon angles the resulting convolution formula, has four convolution variables, two for ISR and FSR and two additional variables for IFI. It is not so handy as the traditional one, because the 5-dimensional (including $\cos \theta$ ) integration has to be done numerically using the $\mathrm{MC}$ method. In Ref. [33] new MC code KKFoam implementing the above calculation was used to cross-check the calculation of the KKMC program in the soft limit, where it should by construction be fully compatible with the Frascati approach.

In the final LEP data analysis [29] the coupling constants of $Z$, its mass and width inside the effective Born (differential) cross section were obtained from the fit to data taken typically only at 3-5 energies. The deconvoluted Born integrated cross section and asymmetries were not really coming directly from the data, but were calculated from the (theo-

\footnotetext{
${ }^{6}$ The ISR radiator function was that of Ref. [31] with $\mathcal{O}\left(\alpha^{1} L_{e}, \alpha^{1}, \alpha^{2} L_{e}^{2}, \alpha^{2} L_{e}, \alpha^{3} L_{e}^{3}\right)$ photonic corrections, contributions from soft fermion pairs and soft photon resummation.
} 
Table 1 Listed are electroweak observables, which are most sensitive to QED effects. Experimental (LEP) errors in the third column are accompanied by an error component in the braces $\{\ldots\}$ induced by QED calculation uncertainties. FCC-ee experimental systematic errors in fourth column are from FCC-ee CDR [2] except $\tau$ polariza- tion [37]. They are all without theoretical uncertainty component. The last column shows the improvement factor in QED theoretical calculations needed in order to be equal to experimental precision of FCC-ee measurements

\begin{tabular}{|c|c|c|c|c|c|}
\hline Observable & Where from & Present (LEP) & FCC stat. & FCC syst & $\frac{\text { Now }}{\text { FCC }}$ \\
\hline$M_{Z}[\mathrm{MeV}]$ & $Z$ linesh. [29] & $91187.5 \pm 2.1\{0.3\}$ & 0.005 & 0.1 & 3 \\
\hline$\Gamma_{Z}[\mathrm{MeV}]$ & Z linesh. [29] & $2495.2 \pm 2.1\{0.2\}$ & 0.008 & 0.1 & 2 \\
\hline$R_{l}^{Z}=\Gamma_{h} / \Gamma_{l}$ & $\sigma\left(M_{Z}\right)[34]$ & $20.767 \pm 0.025\{0.012\}$ & $6 \cdot 10^{-5}$ & $1 \cdot 10^{-3}$ & 12 \\
\hline$\sigma_{\text {had }}^{0}[\mathrm{nb}]$ & $\sigma_{\text {had }}^{0}[29]$ & $41.541 \pm 0.037\{0.025\}$ & $0.1 \cdot 10^{-3}$ & $4 \cdot 10^{-3}$ & 6 \\
\hline$N_{v}$ & $\sigma\left(M_{Z}\right)[29]$ & $2.984 \pm 0.008\{0.006\}$ & $5 \cdot 10^{-6}$ & $1 \cdot 10^{-3}$ & 6 \\
\hline$N_{v}$ & $Z \gamma[35]$ & $2.69 \pm 0.15\{0.06\}$ & $0.8 \cdot 10^{-3}$ & $<10^{-3}$ & 60 \\
\hline $\sin ^{2} \theta_{W}^{\mathrm{eff}} \times 10^{5}$ & $A_{\mathrm{FB}}^{\text {lept. }}[34]$ & $23099 \pm 53\{28\}$ & 0.3 & 0.5 & 55 \\
\hline $\sin ^{2} \theta_{W}^{\mathrm{eff}} \times 10^{5}$ & $\left\langle\mathcal{P}_{\tau}\right\rangle, A_{\mathrm{FB}}^{p o l, \tau}[29]$ & $23159 \pm 41\{12\}$ & 0.6 & $<0.6$ & 20 \\
\hline$M_{W}[\mathrm{MeV}]$ & ADLO [36] & $80376 \pm 33\{6\}$ & 0.5 & 0.3 & 12 \\
\hline$A_{F B, \mu}^{M_{Z} \pm 3.5 \mathrm{GeV}}$ & $\frac{d \sigma}{d \cos \theta}[29]$ & $\pm 0.020\{0.001\}$ & $1.0 \cdot 10^{-5}$ & $0.3 \cdot 10^{-5}$ & 100 \\
\hline
\end{tabular}

retical) fitted effective Born at $s=M_{Z}^{2}$. In other words, the resulting EW pseudo-observables (EPWOs) were encoded in the parameters inside the effective Born, EW pseudoparameters (EWPPs). The definition of the effective Born in Ref. [29] was done at the spin amplitude level; see Eq. (1.34) therein. Could the above scenario be repeated at FCC-ee using an effective Born defined at the amplitude level and factorizing QED at the amplitude level? The detailed numerical studies, as in Ref. [30], at the FCC-ee precision level have to be done from scratch in order to answer this question. In particular an additional uncertainty introduced by partial/incomplete inclusion of the SM effects in EWPOs extracted from the data should be re-examined. In Ref. [4] it was argued that the combined use of more advanced versions of the ZFITTER/TOPAZO programs and of the MC programs of the KKMC class may provide solution.

Summarizing: At the FCC-ee precision IFI requires resummation. Clean factorization into $a$ and a modelindependent (Born-like) part still works at the spin amplitude level, even if it fails (due to non-factorizable contributions) at the amplitude squared level. Such a factorization opens ways to a new more flexible definition of EW pseudo-observables, which would possibly cope with the FCC-ee precision; see Sect. 5.7 for more details.

\section{The most important QED-sensitive experimental observables at LEP and FCC-ee}

The minimum improvement factor to be achieved in the precision of QED perturbative calculations for FCC-ee experiments, in order that these effects are controlled at the level of the pure experimental errors, are shown in the last column of
Table 1. In the third column there we collect the values of the total error of the observables most sensitive to QED effects, as measured at LEP, including also explicitly QED theoretical uncertainty induced in the overall experimental systematic errors. These components are accompanied by the citations of the source papers from which they are taken. The projected much better experimental statistical and systematic errors at FCC-ee experiments are shown in fourth and fifth column. They are taken from Table S.3 in Ref. [2]. As a matter of fact, the actual improvement factor for FCC-ee should be even $2-$ 3 times bigger than the one shown in Table 1, in order to be sure that QED effects are clearly a subdominant component in the corresponding overall systematic error for most of the observables measured at LEP experiments.

The information in Table 1 is the starting point for more detailed discussion in the following sections. The most sensitive to QED effects observables of the FCC-ee experiments listed in the table are total cross section, including low angle Bhabha scattering for luminosity measurement, especially near the $Z$ resonance (for $Z$ mass and width, $Z$ invisible width), cross section of $Z \gamma$ final state ( $Z$ radiative return) above the $Z$ peak, charge asymmetry for leptonic pair final states and spin asymmetry in the $\tau$ pair production. We are analyzing a subset of experimental observables near the $Z$ resonance and $W W$ threshold which are most "vulnerable" to the QED effects - omitting many others.

Let us comment briefly on Table 1, before more detailed discussion in the following sections: QED uncertainty of $M_{Z}$ and $\Gamma_{Z}$ (derived from the lineshape) are taken from Refs. [29, 38]. Huge photonic corrections to the $Z$ lineshape (30\%) in all LEP experimental and theoretical studies are mastered using a formula derived in Ref. [39]. However, the precision of QED corrections in Ref. [29] is dominated by the uncertainty 
of the fermion pair correction of Ref. [40], in spite of the fact that this kind of correction is small by itself. For more discussion, see Sect. 4.1. The QED uncertainty of $R_{Z}^{l}$ is taken from [34] and is mainly due to $t$-channel ${ }^{7}$ obscuring $\Gamma_{e e}$. QED uncertainty of $N_{v}$ from the $Z$ radiative return is from the OPAL paper [35] and its error is enhanced due to the use of the outdated MC programs ${ }^{8}$ with inferior precision $\delta \sigma / \sigma=2 \%$. The factor 60 in last column is therefore a gross overestimate. On the other hand the precision of $N_{v}$ from the peak cross section is limited by the luminosity error $\delta \mathcal{L} / \mathcal{L} \simeq 0.6 \cdot 10^{-3}$, mainly due to QED corrections for the low angle Bhabha luminometer. ${ }^{9}$ Strictly speaking this luminosity error at LEP was mainly of the QCD origin because it was dominated by the vacuum polarization contribution uncertainty due to the experimental error of low energy hadronic data.

The leptonic charge asymmetry of LEP experiments $A_{\mathrm{FB}}^{\text {lept. }}=0.0171(10)$ from Table 2.13 in Ref. [29] translates into an EW mixing angle $\sin ^{2} \theta_{W}^{\text {eff }}=0.23099(53)$; see Table 12.4 in Ref. [34]). It is almost the same as for the muon-pair final state alone. The theoretical error component was estimated in Ref. [29] (Table 2.8) as $\delta A_{\mathrm{FB}}^{0, l}=0.01 \%$ from the difference between ZFITTER and TOPAZ0; see also Ref. [30]. This difference provides for the technical precision of both programs and not what we need, which is uncertainty due to missing higher orders. Such an estimation of the QED perturbative uncertainty of $A_{\mathrm{FB}}$ due to missing higher orders was provided in Ref. [16]. From the comparison of the results of $A_{\mathrm{FB}}\left(M_{Z}\right)$ from KKMC, $\mathcal{O}\left(\alpha^{1}\right)$ KORALZ and ZFITTER (IFI included) in Fig. 28b for the loose cut-off on total photon energy the uncertainty of order $\delta A_{\mathrm{FB}} \simeq 0.05 \%$ seems reasonable. It translates into $\delta \sin ^{2} \theta_{W}^{\text {eff }}=0.57 \cdot \delta A_{\mathrm{FB}}^{0, l} \simeq 0.00027$.

The effective $\sin ^{2} \theta_{W}^{\text {eff }}=0.23159(41)$ in Table 1 from two spin asymmetries, $\left\langle\mathcal{P}_{\tau}\right\rangle$ and $A_{\mathrm{FB}}^{\mathrm{pol}, \tau}$, comes from the LEP summary of Ref. [29] (Sect. 4.4). Both asymmetries have comparable experimental errors. QED effects in the MC simulation of the $\tau$ decays quoted there following Ref. [42] are estimated to contribute $\delta \mathcal{A}_{\tau}=0.0010$ for $\left\langle\mathcal{P}_{\tau}\right\rangle$, which yields $\delta \sin ^{2} \theta_{W}^{\text {eff }}=(1 / 8) \delta \mathcal{A}_{\tau}=0.00012$. For $A_{\mathrm{FB}}^{\mathrm{pol}, \tau}$ the $\mathrm{QED}$ uncertainty according to Ref. [29] is even smaller. The corresponding anticipated FCC-ee experimental statistical and systematic errors are taken from Ref. [2], where it is shown for $A_{\mathrm{FB}}^{\mathrm{pol}, \tau}$. We transformed it into the error of the EW mixing angle using the relation $\delta A_{\mathrm{FB}}^{\mathrm{pol}, \tau} \simeq 6 \cdot \delta \sin ^{2} \theta_{W}^{\text {eff }}$.

The combined LEP measurement of $M_{W}$ in Ref. [36] comes mainly from the $W$ mass reconstruction in the $q \bar{q} q \bar{q}$ and $l \nu_{l} q \bar{q}$ channels. The uncertainty of $6 \mathrm{MeV}$ due to higher

\footnotetext{
7 The $t$-channel subtraction [29] done using ALIBABA program [41] instead of more sophisticated BHWIDE Monte Carlo [27] enhanced unnecessarily this problem.

8 KORALZ and NUNUGPV98 were used instead of KKMC.

9 The LEP era approximate rule for the luminosity error contribution to the error of the number of neutrino species was $\delta N_{v} \simeq 7.5 \delta \mathcal{L} / \mathcal{L}$.
}

order radiative corrections is estimated from comparison of the KandY [14,43] and RACOONWW [15] MC programs.

The LEP experimental error of $A_{\mathrm{FB}, \mu}^{M_{\mathrm{Z}} \pm 3.5 \mathrm{GeV}}$, being $\delta A_{\mathrm{FB}} \sim$ $2 \%$, is mainly statistical, and the QED error estimate $\delta A_{\mathrm{FB}} \sim$ $0.1 \%$ is taken from Refs. [16,44].

Summarizing on Table 1 one may say that the very minimum of the improvement on the QED calculations needed for FCC-ee near the $Z$ resonance and near the $W W$ threshold is typically a factor $\sim 3-60$, with the exception of charge asymmetry, where a bigger improvement $\sim 100$ will be necessary. It is, however, important to stress that in order to get to the same comfortable situation as at LEP, where QED uncertainties (except for the luminosity cross section) were typically at least a factor of 2-3 smaller than the statistical and systematic experimental error, one should really aim at the improvement factors being $\sim 3$ times bigger than these of the last column in Table 1, that is, a factor 10-180 and in the special case of $A_{\mathrm{FB}}$ a factor 300 . With values shown in the last column in Table 1 one will merely get into a rather uncomfortable situation like in the measurement of the luminosity and the total cross section at LEP1, for which the theoretical uncertainty was comparable to the experimental error or even slightly bigger.

\section{Present state of the art in calculating QED effects}

In the following we are going to elaborate in more detail on the present state of art in calculating of the QED effects, as in most cases inherited from the LEP era. It will be done for each observable listed in Table 1.

\subsection{Cross section near the $Z$ resonance: mass and width of $Z$}

The most sizable QED corrections to the total cross section near the $Z$ resonance i.e. the so-called lineshape $(\sim 30 \%)$, are due to multiple photon emissions from the initial $e^{ \pm}$beams (ISR). The basic "effective radiator" formula for the ISR photonic QED corrections was defined in Ref. [39], combining/summarizing results of several other publications.

The master ISR formula of Ref. [39] includes, in addition to the classic $\mathcal{O}\left(\alpha^{1}\right)$ result [45], the relatively simple $\mathcal{O}\left(\alpha^{2} L_{e}^{2}\right)$ contribution [46] and the more elaborate $\mathcal{O}\left(\alpha^{3} L_{e}^{3}\right)$ corrections obtained in the analytical form ${ }^{10}$ in Ref. [48] and cross-checked using dedicated MC program [10]. The next correction $\mathcal{O}\left(\alpha^{4} L_{e}^{4}\right)$ is also available; see Ref. [49]. According to the analysis of Ref. [31], at the LEP precision it is negligible. The ISR formula of Ref. [39] also includes an $\mathcal{O}\left(\alpha^{2} L_{e}^{1}\right)$ photonic contribution from Ref. [8]; see also Refs. [50,51]

$\overline{10}$ This result was later on correctly reproduced in Ref. [47]. 
for corrected $\mathcal{O}\left(\alpha^{2} L_{e}^{0}\right)$ results. In addition to photonic corrections, it includes also the small contribution from fermion pair production of Ref. [10]. It should be remembered that a similar but less precise ISR formula was presented earlier in the pioneering work of Ref. [52]. Both ISR formulas of Refs. [39,52] are employing soft photon resummation. ${ }^{11}$

The master analytical formula for ISR of Ref. [39] was used in the numerical studies of the $Z$ lineshape $(\mid \sqrt{s}-$ $\left.M_{Z} \mid<3 \mathrm{GeV}\right)$ in Refs. [31,38] and in the analysis of all LEP experimental data and all related theoretical studies, as a basic tool for evaluating QED effects in the $Z$ lineshape. In Ref. [31] the total QED uncertainty due to photonic corrections was estimated to be $\delta M_{Z}, \delta \Gamma_{Z} \leq 0.1 \mathrm{MeV}$.

The master convolution formula of Ref. [39] with the ISR radiator function was neglecting contribution from the initial-final state interference (IFI). The uncertainty due to IFI to the lineshape observables was very carefully analyzed in Ref. [38], exploiting older $\mathcal{O}\left(\alpha^{1}\right)$ analytical work (without exponentiation) [53], and results from MC programs with [11] and without [23] soft photon resummation. The IFI correction was found to be of the order $\delta \sigma / \sigma \sim 0.2 \%$ for $\left|\sqrt{s}-M_{Z}\right| \leq 3 \mathrm{GeV}$ to be linearly dependent on $\sqrt{s}$ and causing the shift of $M_{Z}$ of $+0.17 \mathrm{MeV}$ for lepton pair production and $-0.17 \mathrm{MeV}$ for hadronic final states, such that the net contribution of IFI to $M_{Z}$ is small due to the accidental cancellation. Possible uncertainties in the $M_{Z}$ measurement due to IFI-like missing $\mathcal{O}\left(\alpha^{2}\right)$ and $\mathcal{O}\left(\alpha \alpha_{S}\right)$ corrections were discussed in Ref. [38] quantitatively, concluding that the uncertainty of the IFI effect due to higher order corrections is $\delta M_{Z} \simeq 0.1 \mathrm{MeV}$ and $\delta \Gamma_{Z} \simeq 0.1 \mathrm{MeV}$ and of the total cross section at the top of the $Z$ peak is merely $\delta \sigma / \sigma<10^{-4}$. From the analysis of the IFI effect in Ref. [38] it was also clear that the size of the IFI effect and its uncertainty depend quite strongly on the center of the mass (c.m.s.) energy $\sqrt{s}$ and on experimental cut-offs; hence the role of the MC in controlling it was already quite important in the LEP data analysis. A similar analysis of the IFI effect in the lineshape observables was performed in Ref. [30]. It was restricted to pure $\mathcal{O}\left(\alpha^{1}\right)$ QED semi-analytical calculations using ZFITTER[12] and TOPAZ 0[13] programs, without soft photon resummation.

The mass of $Z$ boson comes from fitting of the cross section across the $Z$ resonance (lineshape). According to the final LEP1 and SLD data analysis of Ref. [29], the dominant QED contribution to the lineshape, which translates into $\delta M_{Z} \simeq 0.2 \mathrm{MeV}$ and $\delta \Gamma_{Z} \simeq 0.3 \mathrm{MeV}$, was not from photonic corrections, but rather from the light lepton and quark contributions calculated in Ref. [40]. In the FCC-ee context, this contribution should be cross-checked and carefully reanalyzed.

\footnotetext{
11 YFS resummation of Ref. [39] has turned out to be more effective in resumming higher orders.
}

\subsection{Luminosity measurement}

The overall error of the luminosity measurement quoted by all LEP experiments for LEP1 was below $0.05 \%$, with the best experimental luminosity error $0.034 \%$ being that of the OPAL collaboration. The theoretical prediction of the QED dominated low angle Bhabha (LABH) cross section in all LEP collaborations was based on the calculation from BHLUMI 4.04 Monte Carlo event generator published in Ref. [24]. Its precision, following Ref. [54], was quoted to be $\delta \sigma / \sigma \simeq 0.061 \%$. In fact, the luminosity cross section was the only observable in LEP experiments for which the theoretical uncertainty was bigger than the experimental statistical and systematic error. As seen in Table 2 of Ref. [55] its biggest component was in fact not of the QED origin but due to the vacuum polarization effect, which was calculated using low energy experimental hadronic data. Since in the recent years the error of vacuum polarization contribution was reduced significantly, the updated error of BHLUMI 4.04 predictions is now estimated to be $0.038 \%$ (see the same Table in Ref. [55]) and is now dominated by uncertainties due to missing higher order perturbative QED corrections.

The principal luminosity measurement at FCC-ee will be done using the same type of the low angle Bhabha process as in LEP experiments, using a similar calorimetric detector [56]. Prospects of improving QED predictions for the FCCee luminometry will be discussed in the following.

In Ref. [55]) (Table 3) it was pointed out that due to twice wider angle of the FCC-ee Bhabha luminometer (6486 mrads) than at LEP, the actual theoretical error according to the present state of the art (inherited from LEP) would be in fact $0.090 \%$ due to the bigger $Z$-exchange contribution. However, this error can be reduced to the negligible level already now using the BHWIDE Monte Carlo [27]; see Ref. [55] for the details.

\subsection{EW mixing angle from charge and spin asymmetries at LEP}

All charge and spin asymmetries measured at LEP have their errors dominated by the statistical error [29,34,36,57,58]. Let us characterize briefly these (yet subdominant) QED corrections in the LEP measurements.

Charge and spin asymmetries depend on the ratios of the $Z$ couplings. With the usual simplifications [34] and/or introduction of pseudo-observables (PO) [30], in which QED effects are "deconvoluted", it is convenient to express all charge and spin asymmetries (following the notation of Ref. [29]) in terms of

$$
\mathcal{A}_{f}=\frac{2 g_{V f} / g_{A f}}{1+\left(g_{V f} / g_{A f}\right)^{2}}, \quad \frac{g_{V f}}{g_{A f}}=1-4\left|Q_{f}\right| \sin ^{2} \theta_{\mathrm{eff}}^{f} .
$$


In this work we consider leptonic charge asymmetry and two $\tau$ spin asymmetries

$A_{\mathrm{FB}}^{l}=\frac{3}{4} \mathcal{A}_{e} \mathcal{A}_{l}, \quad A_{\mathrm{FB}}^{\mathrm{pol} \tau}=-\frac{3}{4} \mathcal{A}_{e}, \quad\left\langle\mathcal{P}_{\tau}\right\rangle=-\mathcal{A}_{\tau}$.

The above defines convenient pseudo-observables (pseudoparameters) $\sin ^{2} \theta_{\text {eff }}^{l}, l=e, \mu, \tau$ which allow us to compare information on $Z$ couplings from various asymmetries. Using $\sin ^{2} \theta_{\text {eff }}^{l}=0.2315$ of Table $1\left(A_{l}=0.1472\right)$ and assuming lepton universality, the following simple relations relate uncertainties of asymmetries and of the EW mixing angle:

$\delta \sin ^{2} \theta_{\mathrm{eff}}^{l}=0.5692 \cdot \delta A_{\mathrm{FB}}^{l}, \delta \sin ^{2} \theta_{\mathrm{eff}}^{e}=0.1676 \cdot \delta A_{\mathrm{FB}}^{\mathrm{pol} \tau}$,

$\delta \sin ^{2} \theta_{\text {eff }}^{\tau}=0.1257 \cdot \delta\left\langle\mathcal{P}_{\tau}\right\rangle$.

How big were the estimates of the theoretical uncertainties $\delta A_{\mathrm{FB}}^{l}, \delta A_{\mathrm{FB}}^{\mathrm{pol} \tau}$ and $\delta\left\langle\mathcal{P}_{\tau}\right\rangle$ due to QED effects in the data analysis at LEP near the $Z$ resonance and above?

The first systematic study of the QED uncertainties in $A_{\mathrm{FB}}$ was attempted in the initial LEP workshop [59], in particular the value of the strongly suppressed IFI contribution $A_{\mathrm{FB}}^{\mu, \mathrm{IFI}}=$ $5 \cdot 10^{-4}$ (following Ref. [60]) was established.

As already said, at the $Z$ peak Ref. [29] cites the difference $\delta A_{\mathrm{FB}}^{l}=10^{-4}$ between ZFITTER and TOPAZ0 as a theoretical uncertainty estimate. A closer look into Ref. [30], on which Ref. [29] is based, reveals that the story is more complicated. For IFI switched off, from the ZFITTER--TOPAZ 0 differences and due to the change of the ISR radiation function (from the factorized to additive form) shown in Ref. [30] one may indeed quote $\delta A_{\mathrm{FB}}^{l}\left(M_{Z}\right) \simeq 10^{-4}$ and $\delta A_{\mathrm{FB}}^{l}\left(M_{Z} \pm 3 \mathrm{GeV}\right) \simeq 3 \cdot 10^{-4}$, especially for loose cutoffs. ${ }^{12}$

On the other hand, for IFI switched on, the difference between ZFITTER and TOPAZO in Ref. [30] for realistic cut-offs, within $\left|\sqrt{s}-M_{Z}\right|<3 \mathrm{GeV}$, were quite sizable, of order of a few per mille. ${ }^{13}$ Fortunately, in Ref. [62] the source of these discrepancies between IFI implementation in ZFITTER and TOPAZO were identified and new precision estimates $\delta A_{\mathrm{FB}}^{l}\left(M_{Z}\right)=0.0002$ and $\delta A_{\mathrm{FB}}^{l}\left(M_{Z} \pm 3 \mathrm{GeV}\right)=$ 0.0013 were provided. However, this estimate is not reliable for higher order effects in IFI, because both ZFITTER and TOPAZO implement essentially the same additive combination of the ISR and FSR collinear radiator functions with $\mathcal{O}\left(\alpha^{1}\right)$ results, integrated analytically over a single real photon within some simple cut-offs. Hence the above estimates really represent the technical precision of both programs and not their physical precision.

\footnotetext{
12 Similar earlier study of Ref. [61] based on comparisons of analytical calculations versus of KORALZ Monte Carlo was quoting $\delta A_{\mathrm{FB}}^{l}\left(M_{Z}\right)=$ 0.0005 and $\delta A_{\mathrm{FB}}^{l}\left(M_{Z} \pm 3 \mathrm{GeV}\right)=0.005$.

13 The authors of Ref. [30] were recommending subtraction of IFI using MC programs like KORALZ, with complete $\mathcal{O}\left(\alpha^{1}\right)$.
}

As already noted in Ref. [30] the necessary next step should be simultaneous exponentiation of ISR and IFI. This goal was achieved, almost in parallel with the above work, in the KKMC program [11]. In Ref. [16] the comparison of KKMC with ZFITTER and $\mathcal{O}\left(\alpha^{1}\right)$ KORALZ has shown that indeed for loose cut-offs one may conservatively estimate higher order QED effects to be $\delta A_{\mathrm{FB}}^{\mu}\left(M_{Z}\right)=0.0005$. According to Eq. (4.3) this estimate translates into $\delta \sin ^{2} \theta_{\text {eff }}^{e}=2.8 \cdot 10^{-4}$. This QED uncertainty was about a factor 2 below the LEP experimental precision at the $Z$ peak; see Table 1 .

Later on, at the LEP2 energies near and above the $W W$ threshold the experimental LEP precision of $A_{\mathrm{FB}}$ was worse due to smaller statistics, however, the IFI contribution was again quite important because it was not suppressed. For mild experimental cut-offs it could reach a few percent. The precision of the traditional $\mathcal{O}\left(\alpha^{1}\right)$ calculations of IFI was believed to be $\sim 1 \%$; see Ref. [62]. More systematic studies of the QED uncertainty, including IFI, at LEP2 energies were done in Refs. [16,36,44]. In Ref. [44] the overall QED uncertainty based mainly on comparisons of KKMC with ZFITTER and KORALZ was estimated to be $\delta A_{\mathrm{FB}}^{\mu}\left(M_{Z}\right)=0.004-0.005$ and for the IFI component $\delta A_{\mathrm{FB}}^{\mu, \mathrm{IFI}}\left(M_{Z}\right)<0.003$. The more detailed study of Ref. [16] has concluded that the overall QED uncertainty (including IFI) of the charge asymmetry prediction from $\mathrm{KKMC}$ is $\delta A_{\mathrm{FB}}^{\mu}\left(M_{Z}\right)=0.002$ over the entire LEP2 energy range.

4.4 The invisible $Z$ decay width from cross section and radiative return in LEP experiments

In LEP experiments the measurement of the $Z$ invisible decay width was quantified as the deviation from the Standard Model expectation of the neutrino number and family generation number, $N_{v}=3$. This parameter was measured to be $N_{v}=2.984 \pm 0.008$ [29]. It was deduced mainly from the total cross section at the $Z$ peak $\sigma\left(M_{Z}\right)$. The dominant contribution to its uncertainty was the luminosity error. According to Ref. [29] the luminosity error contributed $\delta N_{v}=7.5 \frac{\delta \mathcal{L}}{\mathcal{L}}$. For $\frac{\delta \mathcal{L}}{\mathcal{L}}=6.1 \cdot 10^{-4}$ it gives $\delta N_{v}=4.6 \cdot 10^{-3}$. At the FCC-ee the above luminosity uncertainty will be again the dominant one. Getting $\delta N_{v}=1 \cdot 10^{-3}$ will require the luminosity error to be improved by a factor $\sim 5$, down to $\frac{\delta \mathcal{L}}{\mathcal{L}}=1 \cdot 10^{-4}$, which looks feasible; see Sect. 5.3.

Another kind of measurement, the so-called $Z$ radiative return (ZRR) was also exploited in LEP experiments; see for instance Ref. [35], where the less precise result $N_{v}=2.69 \pm 0.15$ was quoted. In the ZRR process one is examining the energy distribution of a photon emitted above some minimum angle from the beams, at the c.m.s. energy well above the $Z$ resonance. Such a photon is also seen for $Z$ decaying into neutrinos or other "invisible" particles. The $Z$ resonance peak is seen in the energy distribution 
of the ZRR photon and $N_{v}$ is obtained by means of comparing the integrated cross section of this process with the result of a reference Monte Carlo program. The error of the above LEP measurement $\delta N_{v}=0.15$ was dominated by the statistical error. The QED component of this error was $\delta N_{v}=0.06$, according to $\delta N_{v}=3 \delta \sigma / \sigma$, where $\delta \sigma / \sigma=2 \%$ was the $\mathrm{QED} / \mathrm{SM}$ error attributed to the cross-section calculations of KORALZ [23,63] and NUNUGPV98 [64] programs. Such a precision was then quite satisfactory in view of the large statistical error. The calculation of ZRR using the more advanced KKMC program [11] was not yet available at that time. Another theoretical study was done in Ref. [65]. The improvement at FCC-ee down to $\delta N_{v}=1 \cdot 10^{-3}$ would require $\delta \sigma / \sigma=3 \cdot 10^{-4}$ precision for the MC programs calculating ZRR process (factor $\sim 60$ improvement). However, with the advent of KKMC this factor is clearly an overestimate; see the following discussion.

With FCC-ee precision the error of the luminosity measurement will enter into the game and will have to be improved as well. On the other hand, thanks to the high luminosity of FCC-ee it would be possible to eliminate the dependence on the luminosity error in the ZRR method by means of using the ratio of the photon distribution with invisible $Z$ decay and with $Z$ decaying in the muon pair. Such a method could not be exploited at LEP due to the limited statistics of the ZRR with muon pairs. At FCC-ee it makes sense and the prospects of its precision in terms of $N_{v}$ are discussed in Sect. 5.5.

\subsection{QED in $W$-pair production at LEP, $M_{W}$ measurement}

The role of QED in the LEP2 data analysis near and above the $W W$ threshold was somewhat different from near $Z$ pole - there was no systematic attempt to "deconvolute" universal (process-independent) QED effects and develop the technique of pseudo-observables - QED was usually kept together with pure electroweak corrections and effects due to semi-classical QED interaction of two massive $W$ near the production threshold, the so-called Coulomb effect, had to be included in the game. Generally, there are four classes of QED effects in $e^{+} e^{-} \rightarrow W^{+} W^{-}$or $e^{+} e^{-} \rightarrow 4 f$ process: initial state corrections (ISR), final state corrections (FSR) in the decays of two $W^{ \pm}$, final state Coulomb corrections (FSC) and the so-called non-factorizable interferences ${ }^{14}$ between ISR and FSR in two $W^{ \pm}$decays (IFF). The IFF corrections are suppressed due to relatively long lifetime of $W$ 's. The effects due to ISR are numerically the biggest but easier to control, while the FSR effects can also be quite sizable for typical experimental cut-offs.

\footnotetext{
14 They factorize at the amplitude level but become cumbersome after squaring amplitudes.
}

The experimental error of the measurement of the $W$ boson mass from the total cross section of $e^{+} e^{-} \rightarrow W^{+} W^{-}$ near threshold at LEP2 experiments [36] was $\delta M_{W}=$ $200 \mathrm{MeV}$ due to poor statistics of the data, quoting theoretical error in this measurement as negligible.

The much better experimental precision of $\delta M_{W}=$ $34 \mathrm{MeV}$ was achieved in LEP2 experiments [36] from $W$ mass reconstruction for $l v q \bar{q}$ and $q \bar{q} q \bar{q}$ final states. For this method, the uncertainty of the $\mathcal{O}\left(\alpha^{1}\right)$ theoretical calculations used for $e^{+} e^{-} \rightarrow W^{+} W^{-}$process was estimated in Ref. [36] from the difference between KandY [14,43] and RACOONWW $[15,66]$ programs. In this way, for $l v q \bar{q}$ channel $\delta M_{W}=8 \mathrm{MeV}$ was obtained and for $q \bar{q} q \bar{q}$ it was $\delta M_{W}=5 \mathrm{MeV}$. In particular, the uncertainty due to ISR radiation alone $\delta M_{W}=1 \mathrm{MeV}$ was obtained in Ref. [36] using KandY, RACOONWW and WPHACT [67] by means of switching on/off $\mathcal{O}\left(\alpha^{3} L_{e}^{3}\right)$ ISR contribution.

The general discussion of the theory's issues in the precision SM calculations for the $e^{+} e^{-} \rightarrow W^{+} W^{-}$process can be found in Ref. [68]. However, there was much other work focusing on various specific issues. For instance the detailed analysis of the ISR effects can be found in Ref. [69]. The ISR effect on the total cross section of $e^{+} e^{-} \rightarrow W^{+} W^{-}$near the threshold, at $160 \mathrm{GeV}$ is $-28 \%$ and merely $-7.5 \%$ at $205 \mathrm{GeV}$. However, its uncertainty deduced from switching on/off non-IR $\mathcal{O}\left(L_{e}^{3} \alpha^{3}\right)$ is not bigger than of $2.5 \cdot 10^{-4}$ at $160 \mathrm{GeV}$ and $2.1 \cdot 10^{-4}$ at $205 \mathrm{GeV}$. The entire $-28 \%$ ISR effect at $160 \mathrm{GeV}$ would translate into huge $\delta M_{W} \sim 400 \mathrm{MeV}$, while its uncertainty is worth only $\delta M_{W} \sim 0.2 \mathrm{MeV}$.

Dedicated analysis of the QED and non-QED effects in the $M_{W}$ reconstruction from the final states can be found in Ref. [70]. ${ }^{15}$ The uncertainty due to not included higher order and nonleading ISR effects was estimated in Table 1 of Ref. [70] to be $\delta M_{W}<1 \mathrm{MeV}$, the FSR uncertainty was rated as $\delta M_{W} \sim 2 \mathrm{MeV}$ and the IFF (following Ref. [71]) at $\delta M_{W}<2 \mathrm{MeV}$. The above analysis is based on the results from KandY toolbox of the Monte Carlo and semi-analytical programs [14].

SM calculations for $e^{+} e^{-} \rightarrow 4 f$ process actually used in the LEP2 data analysis were all in form of the Monte Carlo event generators and they included both QED and the remaining EW corrections. In the following we shall provide brief descriptions of them commenting also on how the QED part was organized/implemented. There were two such MC codes with $\mathcal{O}(\alpha)$ corrections to the signal doubly-resonant $\mathrm{CC} 03$ graphs calculated, respectively, in the leading pole (LPA) or double-pole (DPA) approximation and with the tree-level matrix element for the remaining $e^{+} e^{-} \rightarrow 4 f$ contributions:

\footnotetext{
15 A short overview of the experimental methodology is also included there.
} 
- YFSWW3 v.1.16 [25,72] generates the signal process $e^{+} e^{-} \rightarrow W^{+} W^{-} \rightarrow 4 f$ according to the LPA scheme, exploiting the $\mathcal{O}\left(\alpha^{1}\right)$ calculations of Refs. [73-76] and with $W^{ \pm}$decays simulated independently. Multiphotonic radiation for production part, $e^{+} e^{-} \rightarrow W^{+} W^{-}$, is implemented in the YFS framework (EEX scheme). The hard photon ISR is corrected to the $\mathcal{O}\left(L_{e}^{3} \alpha^{3}\right)$. The FSR in two separate $W$-decays is handled by PHOTOS. The KORALW 1.42 [26] code, which simulates the complete $e^{+} e^{-} \rightarrow 4 f$ process at the tree level, has been combined on the event per event basis with the YFSWW3 code. This way a concurrent MC called KandY of Ref. [14] has emerged, which simulates both complete four-fermion final states and includes $\mathcal{O}(\alpha)$ corrections to the Wpair production and decay. The semi-analytical program Korwan for the improved Born approximation (IBA) is included for testing the main MC.

- RACOONWW [15] simulates the complete $e^{+} e^{-} \rightarrow 4 f$ Born-level process, and the single real photon emission process $e^{+} e^{-} \rightarrow 4 f+\gamma$ and implements $\mathcal{O}(\alpha)$ electroweak virtual corrections in the DPA scheme $[77,78]$ exploiting one-loop calculations for on-shell $W W$ production and decay. The ISR radiation is based on the QED collinear structure functions to second order with soft photon exponentiation. It includes also semi-analytical program for the improved Born approximation (IBA) [79], based on CC03 graphs (doubly resonant) and universal corrections to these graphs, i.e. the Coulomb correction at the threshold, the running of effective couplings and collinear ISR.

Both programs include Coulomb corrections for off-shell $W^{ \pm}$bosons. According to expert comparisons of the above two aproaches in Refs. [68,80], DPA and LPA methods ${ }^{16}$ of inserting $\mathcal{O}\left(\alpha^{1}\right) \mathrm{EW}$ corrections into doubly-resonant part of the matrix element in the $e^{+} e^{-} \rightarrow W^{+} W^{-}$process are basicaly equvalent. The main differences are in the implementation of the QED part of the matrix element. The complete $\mathcal{O}(\alpha)$ corrections to $e^{+} e^{-} \rightarrow W^{+} W^{-}$cannot differ - it was checked in Ref. [68] that the difference for the total cross section between implementations in YFSWW3 and RACOONWW of the virtual plus soft corrections in two programs is below $0.01 \%$.

The overall agreement of YFSWW3 and RACOONWW, including all physical effects, is of the order of $0.3 \%$ for the total cross section at $200 \mathrm{GeV}$ [68]. The differences of the $\mathcal{O}\left(\alpha \Gamma_{W} / M_{W}\right)$ are expected due to the inefficiency of DPA/LPA. However, most of these differences arise from the different treatments of the QED corrections in both approaches, so it is interesting to look into them.

\footnotetext{
16 Differences between two variants of LPA are explained in Ref. [43].
}

QED calculations in RACOONWW rely on the massless $e^{+} e^{-} \rightarrow 4 f+\gamma$ matrix element, hence the complete standard full phase space cannot be used. Instead, two methods are used to deal with emission of photons collinear with fermions. In one of them the phase space is "sectorized", i.e. the real photon phase space is restricted with minimum angle of the photon to fermions (also minimum photon energy to exclude IR divergence) and the contribution below the minimum angle is integrated over analytically, recovering correct fermion mass dependence. In another method [81] a QED variant of the Catani-Seymour (CS) subtraction scheme [82] is used. In both these methods collinear ISR or FSR photon angular distributions are integrated over and effective longitudinal momentum distributions (effective radiator functions and/or PDFs) arise. Their original $\mathcal{O}\left(\alpha^{1}\right)$ version is upgraded to include $\mathrm{LO}+\mathrm{NLO}$ corrections up to $\mathcal{O}\left(L_{f}^{3} \alpha^{3}\right)$. Soft photon resummation is also included in the effective radiator functions, but multiphotons are not present explicitly in the $\mathrm{MC}$ events. Non-factorizable interferences between the production and two decays of $W$ s are reproduced up to $\mathcal{O}\left(\alpha^{1}\right)$ (in the soft photon approximation)

The methodology of QED treatment in YFSWW3 or KORALW is quite different from that in RACOONWW. Multiple photons are present explicitly in the MC events. The matrix element is constructed following the EEX variant of the YFS soft photon factorization and resummation. Non-soft higher order LO and NLO collinear photon universal contributions are added to matrix element in the exclusive (unintegrated) form, without collapsing to collinear photon distributions, to a $\delta(\theta)$ function. The multiple photon emission is included in YFSWW3 for ISR and FSR out of $W \mathrm{~s}$, while in KORALW it is restricted to ISR. Single and double photon emissions in the decays of $W$ s are added using PHOTOS[42,83] program. ${ }^{17}$ Non-factorizable interferences between production and two decays of $W$ s are not included. The YFS technique of factorizing and resuming the universal QED corrections employed in YFSWW3 provides a clear separation of the universal QED corrections from the rest of the SM radiative corrections at any order; hence it is a very good candidate for the future inclusions of the non-QED EW corrections beyond the $\mathcal{O}\left(\alpha^{1}\right)$, or in any attempt of better organization (deconvolution) of the existing $\mathcal{O}\left(\alpha^{1}\right)$ calculations.

After LEP2 data were analyzed, the complete $\mathcal{O}(\alpha)$ corrections to $e^{+} e^{-} \rightarrow 4 f$ have been also completed in Refs. [77,78] for the final states without repeated flavors and without CKM-suppressed states. ${ }^{18}$ The comparison of the above new calculation with older LPA/DPA results gives

\footnotetext{
17 Later on in Ref. [84], multiphoton radiation based on the YFS for single $W$ decay was implemented in another MC code WINHAC[84] developed for LHC. This could easily be adapted to the YFSWW 3 code. 18 There is no MC event generator implementing the complete $\mathcal{O}(\alpha)$ correction to the $e^{+} e^{-} \rightarrow 4 f$ process.
} 
new insight into their uncertainty beyond universal QED corrections. Generally these newer calculations confirm the precision estimates of the LPA/DPA approaches of the LEP2 era. Near the $W W$ threshold the difference between the DPA/LPA and the complete four-fermion $\mathcal{O}(\alpha)$ is $\sim 2 \%$ of the fourfermion Born case. Far from the $W W$ threshold the difference of DPA vs. complete four-fermion $\mathcal{O}(\alpha)$ is smaller and drops to below $0.5 \%$, in accordance with the stated precision of the relevant MC codes. (It increases back to $1-2 \%$ at $1-2$ $\mathrm{TeV}$ energies.)

References $[77,78]$ provide also an estimation of the missing higher order EW corrections, with the important conclusion that higher order EW corrections are dominated by the QED contribution $\alpha^{2} \log \left(m_{e}^{2} / s\right)$ and are estimated at $\leq 0.1 \%$ for energies below $500 \mathrm{GeV}$. The other interesting finding is that the contribution of the higher order Coulomb effect is of the order of $0.2 \%$ at the threshold. ${ }^{19}$ The total precision of these four-fermion $\mathcal{O}(\alpha)$ calculations has been estimated by the authors to be a few per mille.

Another calculation specialized to the near $W W$ threshold energy, using the effective field theory (EFT) technique is reported in Refs. [85,86]. The dominant NNLO corrections to four-fermion process $\left(\mu^{-} \bar{v}_{\mu} u \bar{d}\right)$ were calculated using EFT for unstable particles - the best calculation was nick-named $\mathrm{N}^{3 / 2} \mathrm{LO}^{\mathrm{EFT}}$, because in EFT a different expansion parameter (relativistic velocity of $W$ in the $W W$ rest frame) is used to count the strength of particular corrections. The drawback of the EFT method is that it provides the inclusive results only. The effect of these pure $\mathrm{N}^{3 / 2} \mathrm{LO}^{\mathrm{EFT}}$ corrections on the $\mathrm{W}$ mass is estimated as $3 \mathrm{MeV}$.

The complete set of graphs for the $e^{+} e^{-} \rightarrow 4 f$ process at the tree level was implemented in several MC codes [68]. Two of them were used in the actual data analysis of LEP2: standalone version of KORALW [26] and WPHACT [67]. The latter one implements the so-called "Fermion Loop" gauge restoring scheme of Ref. [87].

The present state of the art (mostly inherited from LEP) can be summarized as $0.2 \%$ theoretical precision for the total cross section of the $W$-pair production process in the entire energy range. If statistics was not limited at LEP2, then that would translate into $\delta M_{W} \simeq 3 \mathrm{MeV}$ for the $M_{W}$ measurement from the threshold cross section. Looking at the future needs of the FCC-ee we can see that a factor of 10 improvement in precision is needed relative to the present state of the art. This is a relatively moderate goal as compared to other observables; see Table 1 . We will discuss its feasibility in Sect. 5.6.

\footnotetext{
19 The QCD effects must also be included: $\mathcal{O}\left(\alpha_{S}\right)$ corrections, uncertainties due to matching with parton shower, Bose-Einsein and color reconnections.
}

\section{Prospects of the QED calculation improvements in the FCC-ee measurements}

In contrast to the previous section, where we have elaborated on the present state of the art in the calculations of QED effects for observables selected in Table 1, in the following we shall list all possible developments in QED calculation needed for analyzing future data at FCC-ee. Obviously, this will be to some extent speculative and it is quite probable that we are going to miss some new calculation fronts or methods which will really emerge in the future.

\subsection{Cross section near the $Z$ resonance and mass of $Z$}

As seen in Table 1, the hadronic cross section $\sigma_{\text {had }}^{0}$ at the $Z$ peak was measured with $0.09 \%$ total error with $0.06 \%$ component dominated by the theoretical uncertainty in the luminosity measurement. An anticipated factor of 6 improvement at FCC-ee is due to reduction of the luminosity error down to $0.01 \%$, both in theory and experiment. According to Ref. [31] the uncertainty due to the QED ISR uncertainty in fitting $\sigma_{\text {had }}^{0}$ to the experimental lineshape is $0.02 \%$, while the uncertainty of the IFI contribution [38] is even smaller. The obtained uncertainty was very conservative. For the ISR photonic corrections it was obtained by means of switching on/off parts of the $\mathcal{O}\left(L_{e}^{3} \alpha^{3}\right)$ and $\mathcal{O}\left(L_{e} \alpha^{2}\right)$ components in the effective radiator function and by taking half of them. One should better evaluate analytically known corrections of $\mathcal{O}\left(L_{e}^{4} \alpha^{4}\right)$ [49] and $\mathcal{O}\left(L_{e}^{0} \alpha^{2}\right)$ [8] and estimate the unknown corrections of $\mathcal{O}\left(L_{e}^{2} \alpha^{3}\right)$. Most likely they are at the level of $10^{-5}$. According to Ref. [40] quasi-photonic light fermion contributions (from electron, muon, $\tau$ pairs and light quarks) to the ISR radiator function also contribute in addition $0.02 \%$ to the uncertainty of $\sigma_{\text {had }}^{0}$. Most likely this is an overestimate because this uncertainty is approaching half of the effect itself.

The optimistic point of view might be that, for the hadronic cross section $\sigma_{\text {had }}^{0}$, due to almost $100 \%$ detector acceptance, the known QED calculations are sufficient to reach the $0.01 \%$ precision needed for FCC-ee - only better testing of the existing calculations and better estimates of the missing h.o. corrections are needed. In particular mixed QED-QCD corrections should be re-examined. The improvements on light fermion pair corrections require some effort, but this looks feasible. However, in order to get back to the LEP situation where QED uncertainties were factor $\sim 2-3$ smaller than experimental one then more work would be needed. This would also imply improvements in the luminosity measurement beyond what is described in Sect. 5.3, which will be hard to achieve.

On the other hand, $R_{l}^{Z}=\Gamma_{h} / \Gamma_{l}$ is free of the luminosity problem but more sensitive to QED uncertainties due to relatively complicated acceptance, with the isolation cones 
around beams, more restrictive cut on the total photon energy (or on acollinearity), and the $t$-channel contribution for the $e^{+} e^{-}$final state. The factor 12 improvement in Table 1 for $R_{l}^{Z}$ from $0.60 \%$ down to $0.05 \%$, or even more if the QED uncertainty is to be kept below the experimental error, will require better calculations. The most demanding will be the FSR class of corrections, but ISR and IFI will have to be studied at the level close to the $0.01 \%$ precision level. Semianalytic calculations of the class like ZFITTER [12] and TOPAZ 0 [13] used at LEP will not be sufficient for the task. The upgraded version of KKMC or a similarly accurate Monte Carlo program will have to be developed and used. A dedicated study is needed to determine what level of the improvement of the perturbative calculation is really needed to match the FCC-ee precision of $R_{l}^{Z}$. Most likely it will be one order more across the precision boundaries in Fig. 2 at various perturbative orders in powers of $\alpha$ and mass logarithms for non-soft (IR-finite) QED corrections in the matrix element of the MC program.

The mass of $Z$ comes from fitting the cross section across the $Z$ resonance (lineshape) such that most of the QED effects are removed. It is the hadronic cross section which matters mostly. In the final LEP1 and SLD analysis of Ref. [29] the estimation of QED uncertainties in the measurement of $M_{Z}$ was taken from Ref. [31] for ISR, from [38] for IFI and from [40] for light fermion pairs. According to Ref. [31] the uncertainty due to photonic ISR corrections to light fermion pairs process is negligible because it is weakly dependent on the c.m.s. energy. This statement has to be cross-checked. The effect of IFI according to Ref. [38] is sizable $(\sim 0.17 \mathrm{MeV})$ and its uncertainty induces $\delta M_{Z}, \delta \Gamma_{Z} \leq 0.1 \mathrm{MeV}$ errors. Light fermion pairs also contribute $\delta M_{Z}, \delta \Gamma_{Z} \leq 0.1 \mathrm{MeV}$. Both of these QED uncertainties are therefore of the size of the FCC-ee experimental errors, hence for the comfortable data analysis they should be reduced by at least a factor 23. The IFI effect in the $M_{Z}$ measurement has similar sizes and opposite signs for the leptonic and hadronic final states. It is strongly dependent on the experimental cut-offs. Calculations of $\mathcal{O}\left(\alpha^{1}\right)$ without exponentiation used at LEP for evaluating IFI will not be sufficient at the FCC-ee precision. For extracting $M_{Z}$ and $\Gamma_{Z}$ from $\sigma_{h}(s)$ near the $Z$ peak, further progress in reducing uncertainty of IFI and light fermion pair contributions in $M_{Z}$ beyond the LEP state of the art will be necessary. It is possible that for this particular purpose the hybrid approach of the LEP era combining the use of MC event generators and semi-analytical programs like ZFITTER/TOPAZ0 will still work. However, the alternative approach based entirely on the MC event generators will serve as cross-check and it will also have an advantage to work for more difficult case of charge and spin asymmetries. For the moment KKMC is the leading candidate for further studies of the IFI effects. However, its CEEX matrix element should be upgraded to include $\mathcal{O}\left(\alpha^{3} L_{e}^{3}\right)$ and non-IR parts of
$\mathcal{O}\left(\alpha^{2} L_{e}^{1}\right)$ pentaboxes. For improvements of the light fermion pair contributions one should exploit programs dedicated to four-fermion final states, the same as for the $W W$ production process.

Summarizing, reduction of QED uncertainties in $\sigma_{\text {had }}^{0}$ below $0.1 \%$, in $R_{l}^{Z}$ below $0.05 \%$, in $M_{Z}$ and $\Gamma_{Z}$ below $0.1 \mathrm{MeV}$ is definitely feasible, but it requires more work and improvements of the existing MC and semi-analytical programs. Improvements on light fermion pair contributions seem to be the most urgent.

\subsection{Charge and spin asymmetries at FCC-ee}

Let us concentrate on symmetries at the $Z$ peak, where they will be measured most precisely. As seen in Table 1, the charge and spin symmetries will be measured up to a factor $\sim 50$ more precisely than at LEP, which will require $\mathrm{MC}$ tools for calculating differential distributions with realistic experimental cut-offs including $\mathcal{O}\left(\alpha^{2}\right)$, possibly even $\mathcal{O}\left(\alpha^{3}\right)$, electroweak, QCD and non-universal QED corrections, while resummed QED universal corrections will have to be included at even higher orders. Concerning feasibility of such higher order QED and EW calculations, let us cite statement in the summary of the recent workshop proceeding [4]: "The techniques for higher order SM corrections are basically understood, but not easily worked out or extended ... We anticipate that at the beginning of the FCC-ee campaign of precision measurements, the theory will be precise enough not to limit their physics interpretation."

Presently, the only MC tool (event generator) which provides predictions for all charge and spin asymmetries for arbitrary experimental cut-offs, including $\mathcal{O}\left(\alpha^{1}\right)$ EW corrections, complete $\mathcal{O}\left(\alpha^{2}\right)$ QED corrections and soft-resummed universal QED corrections to infinite order, is the KKMC Monte Carlo [11], at the $Z$ peak and far away from it. Its precision goes far beyond what was needed at LEP, as seen for example in the recent study of the IFI contribution to $A_{\mathrm{FB}}\left(M_{Z} \pm 3.5 \mathrm{GeV}\right)$, where the precision $\delta A_{\mathrm{FB}} \leq 10^{-4}$ was verified using auxiliary calculations. However, for the FCCee experimental precision of asymmetries quoted in Table 1 , the QED part of matrix element has to be upgraded to include the next orders, up to $\mathcal{O}\left(\alpha^{3} L_{f}^{3}\right)$ in the CEEX matrix element, and EW corrections should be upgraded in the MC matrix element to the level of known complete $\mathcal{O}\left(\alpha^{2}\right)$ corrections [88-91]. Needless to say, TAUOLA MC used in KKMC for $\tau$ lepton decays will require additional testing and development. Of course, development from scratch of another MC program of similar quality as KKMC would be of great help in a solid independent validation of the required precision.

On the other hand, let us point out to some important problems with the model-independent representation of the data in a form of simple pseudo-observables like in Table 1, where the information on the fermion couplings to $Z$ extracted from 
charge and spin asymmetries is quantified in terms of a single parameter, $\sin ^{2} \theta_{W}^{\text {eff }}$. At LEP it was possible. The big question is whether for much higher precision at FCC-ee it will be still possible. In the methodology of Ref. [29] a parameter $\sin ^{2} \theta_{W}^{\text {eff }}$ is just in one-to-one correspondence with the ratio of the (real) vector and axial $Z f \bar{f}$ couplings in the effective Born, which was fit to the $e^{+} e^{-} \rightarrow f \bar{f}$ data (taking into account factorizable QED corrections). ${ }^{20}$ Whether this kind of "effective Born" case will be still effective in parametrizing FCC-ee data in the (SM-)independent way is an open question. Some known SM effects could invalidate it, if they are not numerically small as compared to FCC-ee data precision. Most important among them is the $s$-channel non-resonant contribution, which at the $\sqrt{s}=M_{Z}$ drops out because it is almost exactly real, while the $Z$ contribution is purely imaginary. The reduction of the effective c.m.s. energy due to ISR would possibly invalidate that. The electroweak $W W$ and $\gamma Z$ boxes also go beyond the simplistic effective Born ansatz. The $S$-matrix approach, ${ }^{21}$ in which $Z f \bar{f}$ couplings form the residue of the $Z$-pole, provide a nice justification of the effective Born ansatz. These couplings in the $S$-matrix approach have small imaginary parts, which are already included in the LEP definition of the effective Born (see Rq. 1.34 in Ref. [29]). The effects due to EW boxes and imaginary parts of $Z$ couplings were proven in Ref. [30] to be small as compared to LEP data precision, but this might be not true in an FCC-ee environment. Note also that they are unlikely to be affected by new physics.

In particular, although ISR is reducing the c.m.s. energy by $\sim 100 \mathrm{MeV}$, this effect is controlled to within $0.1 \mathrm{MeV}$ [31], hence its effect in charge asymmetry of order $1 \%$ is probably controllable within the FCC-ee data precision. The effect of QED initial-final state interference is suppressed ${ }^{22}$ at the $Z$ peak, $\delta A_{\mathrm{FB}}^{\mathrm{IFI}}\left(M_{Z}\right) \simeq 0.0005$ [59], hence its calculation at the $\mathcal{O}\left(\alpha^{2}\right)$ is probably adequate to take it into account - a better quantitative study of the uncertainty of $A_{\mathrm{FB}}^{\mathrm{IFI}}$ beyond $\mathcal{O}\left(\alpha^{1}\right)$ at the $Z$ peak is definitely needed. Neglecting imaginary parts was found in Ref. [30] to induce $\delta A_{\mathrm{FB}} \simeq 0.15 \%$; hence it will be not negligible for FCC-ee precision at the $\delta A_{\mathrm{FB}} \simeq$ $10^{-5}$ level. On the other hand, EW boxes are a little bit less problematic, as they were found [30] to induce a $\delta A_{\mathrm{FB}} \simeq$ $10^{-4}$ effect only.

Summarizing, in the FCC-ee data analysis it is likely that the LEP-style definition of $\sin ^{2} \theta_{W}^{\text {eff }}$ will have to be either completely abandoned, or replaced by some variant in which all the above effects are not neglected but taken into account. So far, there was no detailed analysis concerning this issue. One may only guess that a model-independent subtraction of

\footnotetext{
${ }^{20}$ According to terminology of Sect. 5.7 it is an example of a "EW pseudo-parameter", EWPP

${ }^{21}$ See Section C.2 in the recent report [4], summarizing on that.

22 Outside the $Z$ resonance $\delta A_{\mathrm{FB}}^{\mathrm{IFI}} \simeq 0.01$.
}

the $s$-channel non-resonant contribution, using data far away from the $Z$ resonance, may still work. Fitting the imaginary parts of the $Z$ couplings in the effective Born to data, that is, treating them as additional pseudo-observables, would be a brave decision. Including $W W$ and $\gamma Z$ boxes and other $\mathcal{O}\left(\alpha^{1}\right)$ EW (QCD) corrections in the effective Born case, i.e. removing them from the data on the way to pseudoobservables would really mean treating them the same way as QED and the major change in the meaning of the EW pseudo-observables (or rather the EW pseudo-parameters). See also the related discussion in Sect. 5.7.

The $\tau$ lepton spin polarization from the $\tau$ pair production at $Z$ is less prone to the ISR QED effects, as it is weakly dependent on $\sqrt{s}$. One specific source of the uncertainties for $\tau$ spin asymmetries is the limited quality of the $\tau$ decays $\tau \rightarrow v_{\tau} \pi, \nu_{\tau} \rho$ used as a spin polarimeter. The effects due to nonperturbative QCD and QED effects in the $\tau$ decays should be controlled better than at LEP. However, it looks like achieving $\delta \sin ^{2} \theta_{W}^{\text {eff }} \simeq 5 \cdot 10^{-5}$ using $\tau$ spin polarization is within the reach of presently available MC tools like TAUOLA and PHOTOS $[42,83]$, provided some extra testing is done [92]. In particular one should "calibrate" $\tau$ decay polarimetric features, implemented in $\tau$ decay simulation MCs, using high statistics $\tau$ decay samples from Belle experiments. ${ }^{23}$ The influence of EW boxes or imaginary parts in the effective $Z$ couplings on the pseudo-observables related to the tau spin asymmetries was not studied in the LEP era, because their precision was statistically limited. More quantitative studies are needed.

Altogether, it is expected that $\sin ^{2} \theta_{W}^{\text {eff }}$ from the $\tau$ lepton spin polarization will be measured at FCC-ee as precisely as from charge asymmetries and will provide a powerful crosscheck on both measurements, due to the very different experimental systematics. The QED induced uncertainties need to be re-examined both in the $\tau$ production and decay processes, but no serious problems are expected.

Just one example of a possible problem: the decay $\tau^{ \pm} \rightarrow$ $\pi^{ \pm} \pi_{0} \nu_{\tau}(\gamma)$ is an efficient $\tau$ spin polarimeter, where the additional photon emission has to be taken into account very precisely. However, the above radiative process has to be distinguished from the cascade decay $\tau^{ \pm} \rightarrow \pi^{ \pm} \omega \nu_{\tau}, \omega \rightarrow \pi^{0} \gamma$, which has a non-negligible combined branching ratio of 0.0015 [93]. The future high precision Belle II data should be used to analyze precisely this and similar effects in the energy spectra of $\tau$ decay product used for measuring $\tau$ polarization at the FCC-ee precision level.

Summarizing, it looks as if in general the main problem is not so much in the better QED and SM calculations of various asymmetries, but rather in the very survival of the methodology of pseudo-observables (pseudo-parameters) used in the

\footnotetext{
23 At the c.m.s. energy of the Belle experiments single taus are not polarized, the tau-pair production allows for this kind of testing.
} 
model-independent representation of data for these asymmetries.

\subsection{Luminosity measurement}

The luminosity measurement at FCC-ee will be based again on the low angle Bhabha process [56], but one should also remember the possible use of the $e^{+} e^{-} \rightarrow \gamma \gamma$ process for the FCC-ee luminometry, which is statistically limited, but not vulnerable to uncertainty due to vacuum polarization; see Sect. C.5 in Ref. [4] for more details.

By the end of the LEP era any substantial improvement of the theoretical prediction for the low angle Bhabha (LABH) process used to determine the LEP luminosity was effectively blocked by the large uncertainty of the vacuum polarization, which was in fact of QCD origin, or more precisely due do experimental errors of the low energy hadronic data. Since then, this uncertainty was reduced by a factor 4 and by the time of FCC-ee experiments another factor 2 is probable. With the present vacuum polarization error, the LEP luminosity error would reduce from $0.061 \%$ down to $0.038 \%$; see Table 2 in Ref. [55]. In this way h.o. perturbative QED components in the uncertainty of the LABH cross section get dominant.

However, any further progress will not be possible without solid control of the so-called technical precision, i.e. any problems due to programming bugs, mistakes in the MC algorithm, and numerical instabilities. The Monte Carlo event generator BHLUMI 4.04 of Ref. [24] was the subject of many internal tests, in particular using elaborate comparisons with semi-analytical calculations in Ref. [94]. These powerful cross-checks were unfortunately limited to not so realistic kinematic cut-offs. The FCC-ee luminometer detector similarly as at LEP will select/detect events in a way which cannot be dealt with using analytic methods. The Monte Carlo is the only way to implement them in the theoretical calculations. Due to all the above features of the luminosity detectors, the only reliable way of controlling technical precision for realistic event selection is to compare calculations from two different MC programs of the comparable quality. In the LEP era there was no other MC program of the same quality as BHLUMI 4.04; hence QED and technical uncertainties were lumped together. Fortunately, it seems that there are two such candidates for the next generation of improved MCs for the LABH process, BHLUMI with the upgraded QED matrix element proposed in Ref. [24] and another MC program BabaYaga [95], developed in recent years, provided its QED matrix element is upgraded.

Assuming that the problems of the low precision of the vacuum polarization and of poor control of the technical precision will be solved, then yet another non-QED component in the budget of the expected theoretical uncertainty of the $\mathrm{LABH}$ process following the LEP era state of the art starts to dominate. As pointed out in Ref. [55], due to the angular range of the FCC-ee luminosity detector being a factor 2 wider, the contribution from the $Z$ exchange near the $Z$ peak will increase by a factor of 4 . If one estimates its uncertainty in the same way as at LEP, then the total luminosity error would jump to $0.097 \%$; see Table 3 in Ref. [55]. However, this uncertainty is rather easy to reduce using the MC program BHWIDE of Ref. [27] developed for the wide angle Bhabha process. In Ref. [55] it was shown that the uncertainty due to the $Z$ exchange can be reduced substantially, perhaps down to $0.001 \%$.

In this context it is worth to mention that vacuum polarization for negative $Q^{2}$ can be obtained directly using the electron-muon scattering process. In the experiment proposed in Ref. [96] with $150 \mathrm{GeV}$ muon beam scattering on a fixed target electron it will be possible to measure directly QED effective coupling within the range $0>Q^{2} \geq$ $-0.140 \mathrm{GeV}^{2}$. However, SM calculations for FCC-ee processes (low angle Bhabha) will need vacuum polarization for $\left|Q^{2}\right| \geq 1 \mathrm{GeV}^{2}$.

The path of reduction of uncertainties due to pure QED corrections was also described in Ref. [55]. In particular it was pointed out that the dominant $\mathcal{O}\left(\alpha^{2} L_{e}\right)$ correction not included in BHLUMI 4.04 is already known since a long time ago [97] and it can be added to the BHLUMI matrix element rather easily. However, the most elegant solution would be to implement in BHLUMI the QED matrix element with the same type of CEEX resummation as in KKMC, including in addition the $\mathcal{O}\left(\alpha^{3} L_{e}^{3}\right)$ corrections. According to Ref. [55], this would reduce the uncertainties due to all photonic corrections to the level below $10^{-4}$.

Let us note that subleading $\mathcal{O}\left(\alpha^{2}\right)$ QED corrections including non-logarithmic terms to low angle Bhabha were also calculated in Refs. [98-100].

The quasi-photonic corrections due to emission of the light fermion pairs are small but difficult to master. Reduction of their uncertainty below $10^{-4}$ level will require a special effort and most likely development of another dedicated MC program. The first step in this direction was done in Refs. [101,102].

It is quite likely that in FCC-ee times one may be in the situation in which the dominant contributions to the luminosity uncertainty $\sim 0.01 \%$ will be the experimental one from detector and indirectly the experimental one from the vacuum polarization (QCD), while the pure QED contribution will be again subdominant.

Summarizing, according to Ref. [27], the total uncertainty of the theoretical prediction for the luminosity cross section will be at the $10^{-4}$ level, that is, a factor $\sim 10$ better that at LEP times. This will require an upgraded matrix element in BHLUMI to $\mathcal{O}\left(\alpha^{2}\right)$ CEEX level, development of another MC program of comparable class to control technical pre- 
cision and further reduction (a factor of 2) of the vacuum polarization error contribution.

\subsection{Measurement of $\alpha_{Q E D}\left(M_{Z}\right)$ using $A_{\mathrm{FB}}$ near $Z$ resonance}

The idea of using precision measurement of the charge asymmetry $A_{\mathrm{FB}}$ near the $Z$ resonance in order to extract the QED coupling constant at the electroweak scale $\alpha_{\mathrm{QED}}\left(M_{Z}\right)$ was proposed quite recently in Ref. [103]. It is an alternative to the current method calculating $\alpha_{\mathrm{QED}}\left(M_{Z}\right)$ using as an input very precisely known $\alpha_{\mathrm{QED}}(0)$ and the low energy hadronic cross section as an input to the dispersion relations. The new method requires the charge asymmetry to be measured with the precision $\delta A_{\mathrm{FB}} \simeq 10^{-5}$ at $\sqrt{s_{ \pm}} \simeq M_{Z} \pm 3.5 \mathrm{GeV}$. According to Ref. [103] both statistical and experimental systematic error can be reduced to this fantastic low level, a factor $\sim 200$ better than what we have seen in LEP experimental results.

Concerning QED uncertainties, it was argued in Ref. [103] that because both ISR and IFI corrections do not change sign across the $Z$ peak, they will cancel in the difference $A_{\mathrm{FB}}\left(s_{+}\right)-A_{\mathrm{FB}}\left(s_{-}\right)$. However, in the analysis of Ref. [33] based on the numerical results of KKMC it was shown that although such a cancellation is present, nevertheless it is not perfect and the remaining net effect in the difference is of order $\sim 1 \%$. On the other hand, quite fortunately, the QED effects near the $Z$ resonance, for a relatively sharp cut-off on the total photon energy $E_{\gamma} \leq 0.02 E_{\text {beam, }}$ are governed by soft photon emissions. Therefore, the resummation of higher order corrections using the soft photon approximation, which is the basis of the CEEX matrix element of $\mathrm{KKMC}$, is very efficient, not only for the total cross section, but also for the charge asymmetry.

In the study of Ref. [33] the results of KKMC for $A_{\mathrm{FB}}\left(s_{ \pm}\right)$ and their difference were validated by means of comparing them with the newly developed MC program KKFoam with partial analytical integration and soft photon resummation. It was shown that the predictions of KKMC can be trusted down to $\delta A_{\mathrm{FB}}\left(s_{ \pm}\right) \sim 10^{-4}$ precision. Also, some additional internal tests of $\mathrm{KKMC}$ indicate that the level of $\delta A_{\mathrm{FB}}\left(s_{ \pm}\right) \sim 10^{-5}$ is attainable in the results of $\mathrm{KKMC}$ for the difference $A_{\mathrm{FB}}\left(s_{+}\right)-A_{\mathrm{FB}}\left(s_{-}\right)$. The above results have to be consolidated, but they indicate that thanks to the enormous power of the soft photon resummation at the amplitude level developed in Ref. [16] the improvement of the QED prediction for $A_{\mathrm{FB}}$ near the $Z$ resonance by the factor 200 needed for the FCC-ee experiments looks feasible. The above analysis concentrates on the pure photonic corrections to $A_{\mathrm{FB}}$, and many other corrections like light pair emissions, QCD, and electroweak corrections in this extreme precision regime will have to be analyzed very carefully. In particular it should be checked to what extent all these corrections/effects cancel in the difference $A_{\mathrm{FB}}\left(s_{+}\right)-A_{\mathrm{FB}}\left(s_{-}\right)$.

Summarizing, the present status of the QED uncertainties (mainly due to IFI) is that they can be controlled in $A_{\mathrm{FB}}\left(s_{+}\right)-A_{\mathrm{FB}}\left(s_{-}\right)$and in $\alpha_{\mathrm{QED}}\left(M_{Z}\right)$ down to the $\sim 10^{-4}$ level. Another factor 10 improvement will require hard work but according to the preliminary study in Ref. [16] it looks feasible.

\subsection{Invisible $Z$ decay width from cross section and radiative return}

In the determination of $N_{v}$ from the peak cross section the error of the luminosity is the main obstacle in the precision improvement. Using the approximate rule $\delta N_{v}=7.5 \frac{\delta \mathcal{L}}{\mathcal{L}}$ of Ref. [29] we estimate that in order to get $\delta N_{v} \simeq 10^{-3}$ the luminosity relative error has to be improved from the present $\delta \mathcal{L} / \mathcal{L} \simeq 5 \cdot 10^{-4}$ down to $1 \cdot 10^{-4}$. As discussed in Ref. [55] it is feasible, provided that the QED matrix element in BHLUMI is upgraded to the $\mathcal{O}\left(\alpha^{2}\right)$ CEEX level, similarly to $\mathrm{KKMC}$, and the vacuum polarization at $t=-1 \mathrm{GeV}^{2}$ is improved by another factor 2. The error of $\sigma\left(M_{Z}\right)$ due to missing higher orders in the QED ISR calculations (including light fermion pairs emission) was conservatively estimated in Ref. [31] to be $\delta \sigma / \sigma\left(M_{Z}\right) \simeq 2 \cdot 10^{-4}$. The factor 2 improvement is probably obtainable by means of a careful re-examination of the existing calculations. Reducing the experimental systematic error to the same level is of course an independent important issue.

The prospects of reducing the QED uncertainty in the $Z$ radiative return (ZRR) process are discussed in Ref. [104]. Here we only summarize the main points.

First of all, in the direct determination of $N_{v}$ from the ZRR neutrino-like cross section one has to take into account that the QED matrix element of KKMC is much better than that of KORALZ [105] and NUNUGPV98 [64] used at LEP. Nevertheless, due to selecting an event with one real photon, one is effectively losing one perturbative order and it is effectively "downgraded" from the complete $\mathcal{O}\left(\alpha^{2}\right)$ exp. to $\mathcal{O}\left(\alpha^{1}\right)$ exp. Validating the precision of the KKMC for the ZRR process is not yet completed - an optimistic estimate of Ref. [104] for the photonic uncertainty of the ZRR process is quite promising, $\delta N_{\nu} / N_{v} \simeq 2.4 \cdot 10^{-5}$ for $105 \mathrm{GeV}^{24}$ and $\delta N_{v} / N_{v} \simeq 2.2 \cdot 10^{-4}$ for $161 \mathrm{GeV}$. The present error due to the luminosity $\delta \mathcal{L} / \mathcal{L} \simeq 5 \cdot 10^{-4}$ [29] implies $\delta N_{v} / N_{v}=$ $3 \frac{\delta \mathcal{L}}{\mathcal{L}} \simeq 1.5 \cdot 10^{-3}$ and it would dominate, although its reduction by a factor of 2-5 according to Ref. [55] is feasible. More conservatively, for full exploitation of the FCC-ee precision it would be recommended to develop a new dedicated MC program with the complete $\mathcal{O}\left(\alpha^{2}\right)$ exp. matrix element for

\footnotetext{
$\overline{{ }^{24} \text { The energy }} \sqrt{s}=105 \mathrm{GeV}$ is not included in the FCC-ee operational mode.
} 
the ZRR neutrino-like process. In the mean time one should validate more precisely the predictions of KKMC for ZRR by means of a comparison with the semi-analytical programs based on collinear structure functions similar to KKsem [16] or NUNUGPV9 8 [64].

In the method of extracting $N_{\nu}$ from the ratio of the ZRR photon spectrum for the invisible $Z$ decay and $Z$ decaying into muon pairs, the luminosity will cancel out. In addition the effect of the ISR will also cancel quite precisely as well, as shown in Ref. [104]. On the other hand, due to the cutoff on the photon angle a sizable QED FSR effect in the muon-pair process will remain in the ratio; see [104]. Note that the contribution from the IFI is also sizable, but does not contribute much to the integrated cross section because it changes sign across the $Z$ peak in the photon spectrum. Consequently, a high quality MC program for predicting ZRR with muon pairs will be mandatory. KKMC will be obviously helpful, provided its validation using auxiliary programs is pursued, but ultimately a genuine $\mathcal{O}\left(\alpha^{2}\right)$ exp. for the muonic ZRR is highly desirable.

Summarizing, the optimistic estimate of the photonic QED correction in Ref. [104], based on the internal crosschecks of KKMC, (dominated by the FSR effect in the muonpair ZRR) is $\delta N_{v} \simeq 0.9 \cdot 10^{-3}$, for both c.m.s. energies, $105 \mathrm{GeV}$ and $161 \mathrm{GeV}$. It would be necessary to develop a new MC program dedicated to the ZRR process in order to cross-check the above result and gain another factor 3, needed for pushing the uncertainty of QED corrections to a subdominant level.

\subsection{Cross section near the $W W$ threshold $\left(M_{W}\right.$ measurement)}

Until recently there was no quantitative reliable estimate of the experimental precision of the $M_{W}$ measurement at FCCee from the $W$ mass reconstruction using final state quarks and leptons. ${ }^{25}$ According to recent studies presented at the FCC Week 2019 [106] both types of measurements, from final state mass reconstruction and from the threshold cross section, will provide a precision within a similar range of $0.28-0.45 \mathrm{MeV}$. In the following we shall refer mainly to the simpler measurement of $M_{W}$ at FCC-ee from the $W$-pair threshold cross section.

The mass of the $W$ boson can be determined very precisely from the value of the total cross section near the threshold of the $e^{+} e^{-} \rightarrow W^{+} W^{-}$process, at the c.m.s. energy where the cross section is most sensitive to $M_{W}$, which is $\sim 162.5 \mathrm{GeV}$ [3]. (Similarly, the best c.m.s. energy for a $\Gamma_{W}$ determination is $157.5 \mathrm{GeV}$.) Knowing the accuracy of the cross section, the precision of the $W$ mass determined from the threshold scan

25 Theoretical uncertainties in this method were estimated in Ref. [36] to be $5-10 \mathrm{MeV}$, while in Ref. [70] they were estimated as $\leq 2 \mathrm{MeV}$. can be determined approximately from the following relation [107]:

$\Delta M_{W}=\Delta \sigma_{W W}\left|\frac{\mathrm{d} M_{W}}{\mathrm{~d} \sigma_{W W}}\right|=\sqrt{\sigma_{W W}}\left|\frac{\mathrm{d} M_{W}}{\mathrm{~d} \sigma_{W W}}\right| \frac{\sqrt{\sigma_{W W}}}{\sqrt{N}}$,

where $\Delta \sigma_{W W}=\frac{\sigma_{W W}}{\sqrt{N}}$ is the statistical error. The sensitivity factor $\sqrt{\sigma_{W W}}\left|\frac{\mathrm{d} M_{W}}{\mathrm{~d} \sigma_{W W}}\right|$ is estimated in [107] to be $0.91 \mathrm{GeV} / \sqrt{\mathrm{pb}}$ at the $W W$ threshold.

The physics goals of FCC-ee are set to 10/ab and $3 \times$ $10^{7}$ events at the $W W$ threshold [108]. For example, for the cross section $\sigma_{W W} \simeq 3 \mathrm{pb}$ and the accuracy of the total cross section $\frac{\Delta \sigma_{W W}}{\sigma_{W W}}=1 / \sqrt{3 \times 10^{7}}=0.02 \%$ the resulting accuracy of the $W$ mass would be

$$
\begin{aligned}
\Delta M_{W} & =0.91 \frac{\mathrm{GeV}}{\sqrt{\mathrm{pb}}} \frac{\sqrt{3 \mathrm{pb}}}{\sqrt{3 \times 10^{7}}} \\
& \simeq 0.3 \mathrm{MeV}=3.6 \times 10^{-6} M_{W} .
\end{aligned}
$$

The ultimate goal of the measurement of the total cross section at the $W W$ threshold at the FCC-ee is the precision of $0.02 \%$. This requires at least a factor 10 improvement of the theory's precision for the SM calculation of the $W$-pair production near the threshold.

The first step in this direction is obvious - a MC program with the complete $\mathcal{O}(\alpha)$ EW corrections to $e^{+} e^{-} \rightarrow 4 f$ process must be available. This task is definitely doable since the analytical/numerical calculations already exist. That would consolidate the EW precision at the level of $0.2 \%$. In order to reduce the precision further a $\mathcal{O}\left(\alpha^{2}\right)$ calculation for the doubly-resonant $e^{+} e^{-} \rightarrow W^{+} W^{-}$process is needed, with some clever strategies inspired by EFT near the threshold.

A complete EW $\mathcal{O}\left(\alpha^{2}\right)$ calculation for the $e^{+} e^{-} \rightarrow 4 f$ and $\mathcal{O}\left(\alpha^{3}\right)$ for the $e^{+} e^{-} \rightarrow W^{+} W^{-}$process seems to be very hard to do, but one may try to estimate uncertainties in the case it is not available. Firstly, let us try to estimate an error due to the neglected $\mathcal{O}\left(\alpha^{3}\right)$ EW corrections. There are no tools to determine their actual size. However, one can do naive scaling based on the lower order corrections. Namely, if at $161 \mathrm{GeV} \mathcal{O}(\alpha) \sim 2 \%$ of Born (ISR excluded) and $\mathcal{O}\left(\alpha^{2}\right) \leq 0.2 \%$ of Born, i.e. there is a factor of 10 suppression between them, then we can assume the same factor of 10 between $\mathcal{O}\left(\alpha^{2}\right)$ and $\mathcal{O}\left(\alpha^{3}\right)$ and we end up at $\mathcal{O}\left(\alpha^{3}\right) \leq 0.02 \%$ of Born, which shows that the $\mathcal{O}\left(\alpha^{3}\right)$ contribution should be just negligible.

Since an $\mathcal{O}\left(\alpha^{2}\right)$ complete calculation for the $e^{+} e^{-} \rightarrow 4 f$ process is a difficult challenge, one should consider another, simpler scheme. This scheme would mimic the approach from LEP2 based on calculating separately the $\mathcal{O}\left(\alpha^{2}\right)$ corrections to the production and decay of $W$-pair, i.e. to $e^{+} e^{-} \rightarrow$ 
$W^{+} W^{-}$and then $W \rightarrow 2 f$ process. ${ }^{26}$ Similarly to LEP2, but at one order higher, the above calculations would be combined with the existing $\mathcal{O}\left(\alpha^{1}\right)$ complete calculation for the $e^{+} e^{-} \rightarrow 4 f$ process. What can we say about the precision of such an approach? As earlier, we can only do a naive scaling from the lower order. Namely, analyzing the single-pole (SP) and double-pole (DP) contributions at $\mathcal{O}(\alpha)$ we find from $[77,78]$ that at $161 \mathrm{GeV}$ we can estimate the $\mathcal{O}(\alpha)_{S P}$ as

$$
\begin{aligned}
\mathcal{O}(\alpha)_{S P} & \sim\left(\mathcal{O}(\alpha)_{4 f}-\mathcal{O}(\alpha)_{D P}\right) \sim 2 \% \text { of Born } \\
& \sim 8 \% \text { of } \mathcal{O}(\alpha)_{D P} .
\end{aligned}
$$

If we now assume, as argued earlier, that we have $\mathcal{O}\left(\alpha^{2}\right) \leq$ $0.2 \%$ of the Born calculation and we extrapolate from the first order that $\mathcal{O}\left(\alpha^{2}\right)_{S P} \sim 8 \% \mathcal{O}\left(\alpha^{2}\right)$, then we arrive at $\mathcal{O}\left(\alpha^{2}\right)_{S P} \sim 0.016 \%$, within the targeted precision of $0.02 \%$.

Near the threshold, where the split into DP+SP+NP, using a decomposition into powers of $\Gamma_{W} / M_{W}$ becomes inefficient, one should try to exploit expansion in powers of $\beta, \beta^{2}=\left(s-4 M_{W}^{2}\right) / 4 M_{W}^{2}$, as in EFT calculations of Refs. [85,86], see also the review in Ref. [6], but for the traditional diagrammatic calculation within the standard phase space, which can be implemented within the MC event generator. This may reduce significantly the number of diagrams in the $\mathcal{O}\left(\alpha^{2}\right)$ for $e^{+} e^{-} \rightarrow 4 f$ to a manageable subset.

In Sect. 4.5 it was underlined that the implementation of the QED universal corrections in RACOONWW on one hand and in KORALW and YFSWW3 on the other hand was very different. The immediate question is whether these approaches can be easily extended to new Monte Carlo event generators including a complete $\mathcal{O}\left(\alpha^{2}\right)$ for $e^{+} e^{-} \rightarrow W^{+} W^{-}$matrix element or any complete or partly complete $\mathcal{O}\left(\alpha^{2}\right)$ one for $e^{+} e^{-} \rightarrow 4 f$ ? It looks as if it will be very hard to extend the methodology of RACOONWW to $\mathcal{O}\left(\alpha^{2}\right)$ - the subtraction technique of Catani-Seymour is limited to $\mathcal{O}\left(\alpha^{1}\right)$ and the "sectorization" technique becomes very complicated beyond $\mathcal{O}\left(\alpha^{1}\right)$. On the other hand, an example of the MC, KKMC program, with complete $\mathcal{O}\left(\alpha^{2}\right)$ QED for the $e^{+} e^{-} \rightarrow 2 f$ process exists already, and a YFS-inspired CEEX technique $[11,16]$ of factorizing and resumming a universal QED correction within the $\mathrm{MC}$ event generator will work at the practical level both for the $e^{+} e^{-} \rightarrow W^{+} W^{-}$and the $e^{+} e^{-} \rightarrow 4 f$ processes. In particular, the CEEX technique is very well suited for implementing "non-factorizable" interferences between the $W^{+} W^{-}$production process and two $W$ decays and between the two $W$ decays as well. It extends the exponentiation of soft photons of Ref. [17]. In the classical approach one exponentiates only interferences and emissions from external legs.

\footnotetext{
${ }^{26}$ One could profit from two-loop corrections to the muon decay which are known since long [109].
}

In the new CEEX-style scheme sketched in Refs. [110, 111] also photon radiation including interferences from the internal charged $\mathrm{W}$ bosons is exponentiated. One may ask whether it makes sense as there are no related infrared singularities present. The answer is yes, because the $W \mathrm{~s}$ can be treated as almost stable particles. The main features of the above scheme are the following:

- The missing interferences for production-decay and decay-decay to the $W W$ graphs are included to all orders in the soft approximation.

- The shift in kinematics due to interferences/emissions (recoil) in the $\mathrm{W}$ propagators is properly described.

- Higher order corrections can easily be added to the nonsoft functions ${ }^{27}$ resulting from the IR subtractions.

The above scheme would work for single- $W$ or $Z Z$ pair production as well. The implementation may start with the simpler EEX-type scheme, already partly implemented for $e^{+} e^{-} \rightarrow W^{+} W^{-}$in YFSWW 3 and for $W^{ \pm}$decays in WINHAC MC [84], without production-decay and decay-decay interference. The CEEX scheme would be implemented in the next step by means of reweighting MC events with multiplicative weight, similarly to KKMC. The above would take care of the doubly-resonant part of $e^{+} e^{-} \rightarrow 4 f$. The implementation of the CEEX matrix element for the singleresonant part would be straightforward. For the non-resonant part one probably needs only CEEX for ISR and some crude FSR implementation, neglecting IFF interference.

Summarizing, the precision of the $W W$ legacy MC codes from LEP2 is $2 \%$ at the threshold, as follows from the direct comparison with the complete calculation $[77,78]$. The inclusion of this complete $\mathcal{O}(\alpha)$ calculation would improve the EW precision to the level of $0.2 \%$, as follows from the dominant $\mathcal{O}\left(\alpha^{2}\right)$ calculation of $[85,86]$ and the estimates in [77,78]. To achieve $0.02 \% \mathrm{EW}$ precision of the cross section one has to compute and implement the $\mathcal{O}\left(\alpha^{2}\right)$ EW corrections. We argued that it could be enough to calculate them in double/leading pole approximation, supported by the methods of near-threshold improvements of Refs. [85,86]. The exponentiation of real and virtual radiation from intermediate $W$ s proposed in Ref. [110] can further improve the precision. The QCD corrections have to be analyzed and included as well. This way the overall SM precision tag $\sim 10^{-4}$ for the $e^{+} e^{-} \rightarrow W^{+} W^{-}$process seems feasible. The YFSWW+KORALW approach looks like a good starting point for the above developments. The same improvements

\footnotetext{
27 Non-soft functions at $\mathcal{O}\left(\alpha^{1}\right)$ for double-resonant and single-resonat parts of the $e^{+} e^{-} \rightarrow 4 f$ process are defined in Sects. 4.2 and 4.3 of Ref. [111]. For the non-resonant part they are defined the same way as in the standard YFS/CEEX scheme; see also Sect. C.2.7 in Ref. [4].
} 
Fig. 3 Scheme of construction and the use of EWPO/EWPP at FCC-ee

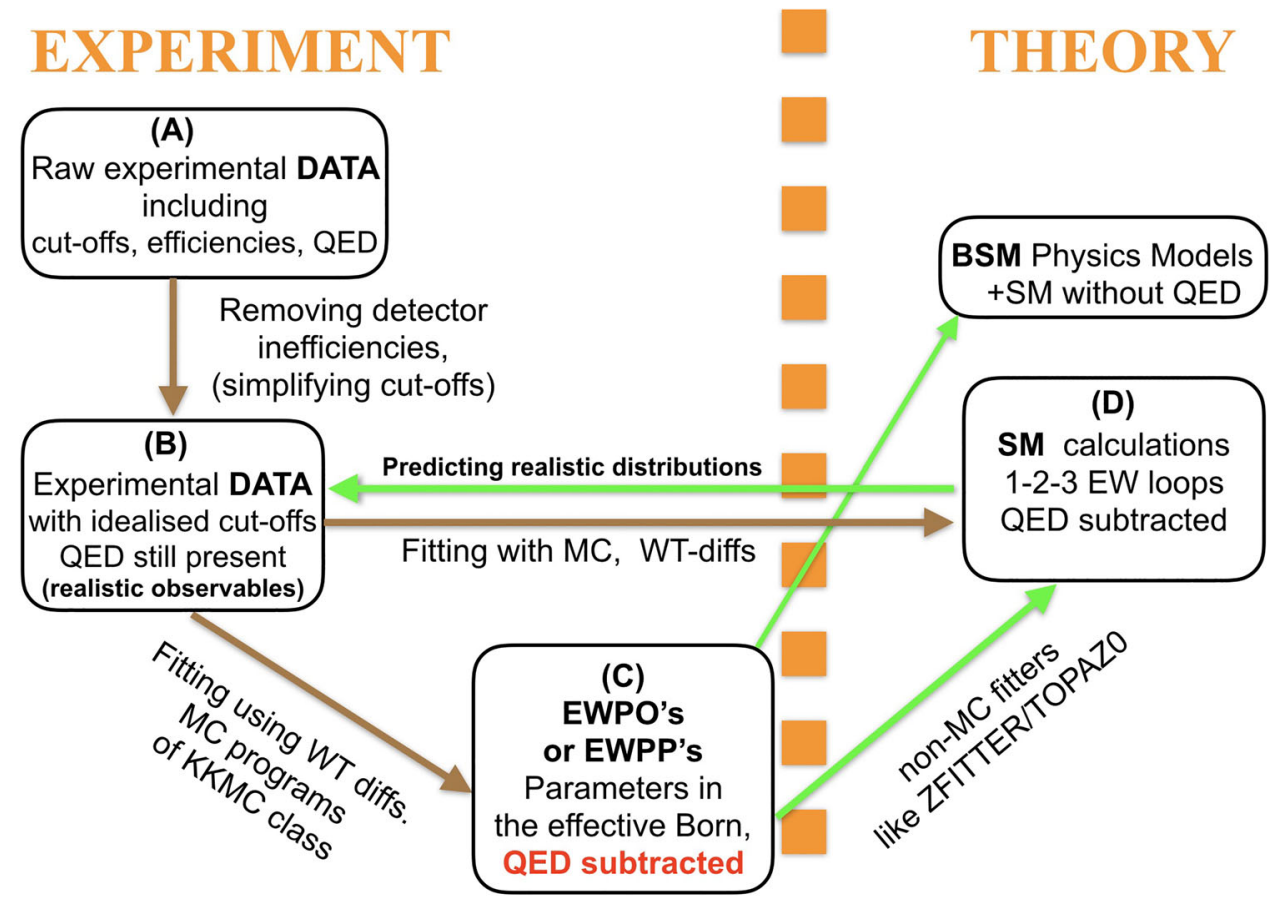

of the theoretical calculations and MC programs are essential for $M_{W}$ measurements using final state mass reconstruction.

\subsection{New ideas on pseudo-observables at FCC-ee}

The system of electroweak pseudo-observable (EWPOs) used in final analysis of LEP data near $Z$ resonance was forged in the interaction between theorists and experimentalists $[29,30]$. As stated before, it may not work at the higher experimental precision of FCC-ee. The authors of Sect. C2 of Ref. [4] propose to modify the EWPO scheme of LEP to the FCC-ee level. The main aim is to preserve its fundamental feature, that is, to provide a flexible link between data and theory. EWPOs should provide model-independent encapsulation (representation) of experimental data, where model independence means removing from the data technical details of the detector (inefficiencies), kinematic cut-offs and large universal QED corrections.

Let us explain this new modified scheme of EWPOs following its graphical representation in Fig. 3. Similarly to the LEP case, the EWPOs are encoded in the parameters of some effective Born quantities (couplings, masses) such that EWPOs like cross sections, asymmetries, and partial widths are in one-to-one correspondence with these parameters, which are called EW pseudo-parameters (EWPPs). The critical question is whether one may factorize-off and remove QED corrections at the FCC-ee precision level on the way from raw experimental data to EWPOs/EWPPs, $(A) \rightarrow(C)$ in Fig. 3.
As indicated in the previous section, the QED deconvolution of LEP, which was done at the amplitude squared level and ignoring non-factorizable IFI effects, will be unable to meet FCC-ee precision criteria. However, the QED factorization at the amplitude level has the extra power for the task. The price to pay is that it cannot be done analytically, but has to be implemented using a numerical resummation within the Monte Carlo. The corresponding transformation of data $(B) \rightarrow(C)$ in Fig. 3 was done in the LEP scenario using ZFITTER/TOPAZO programs and in the proposed scheme the fitting parameters in the effective Born approach would be done by the Monte Carlo programs. An additional advantage is the full flexibility concerning the choice of experimental cut-offs at the stages $(A)$ or $(B)$ in Fig. 3. In addition, the MC program will have not only effective Born amplitudes convoluted with multiple photon emissions, but also $\mathcal{O}\left(\alpha^{1}\right)$ or even $\mathcal{O}\left(\alpha^{2}\right)$ non-IR electroweak corrections; thus it will be able to fit directly data to SM predictions, as indicated in $(B) \rightarrow(D)$ in Fig. 3. This is important, because what we want for new EWPOs/EWPPs is the possibility of fitting data to SM, $(C) \rightarrow(D)$ in Fig. 3, as in the case of the LEP scenario.

A direct fit of data to the SM predictions, $(B) \rightarrow(D)$, will provide a cross-check on the precision loss in the desired two-step scenario $(B) \rightarrow(C) \rightarrow(D)$. Another powerful cross-check done in Ref. [30] was to close the loop $(B) \rightarrow$ $(C) \rightarrow(D) \rightarrow(B)$. Cross-checks of this kind were also done in Ref. [29] in the final LEP data analysis. Obviously, the role of the $\mathrm{MC}$ in the proposed scheme is more important than at the LEP scenario. It will also require implementing 
Table 2 Comparing experimental and theoretical errors at LEP and FCC-ee as in Table 1. The third column shows LEP experimental error together with uncertainty induced by QED and the fourth column shows anticipated FCC-ee experimental statistical [systematic] errors.
An additional factor $\times 3$ in the fifth column (fourth in Table 1) reflects what is needed for QED effects to be subdominant. Rating from * to ${ }^{\star \star \star}$ marks whether the needed improvement is relatively straightforward, difficult or very difficult to achieve

\begin{tabular}{|c|c|c|c|c|c|}
\hline Observable & $\begin{array}{l}\text { Source } \\
\text { LEP }\end{array}$ & $\begin{array}{l}\text { Err. }\{Q E D\} \\
\text { LEP }\end{array}$ & $\begin{array}{l}\text { Stat[Syst] } \\
\text { FCC-ee }\end{array}$ & $\frac{\text { LEP }}{\text { FCC-ee }}$ & $\begin{array}{l}\text { main development } \\
\text { to be done }\end{array}$ \\
\hline$M_{Z}[\mathrm{MeV}]$ & $Z$ linesh. & $2.1\{0.3\}$ & $0.005[0.1]$ & $3 \times 3^{\star}$ & Light fermion pairs \\
\hline$\Gamma_{Z}[\mathrm{MeV}]$ & $Z$ linesh. & $2.1\{0.2\}$ & $0.008[0.1]$ & $2 \times 3^{\star}$ & Fermion pairs \\
\hline$R_{l}^{Z} \times 10^{3}$ & $\sigma\left(M_{Z}\right)$ & $25\{12\}$ & $0.06[1.0]$ & $12 \times 3^{\star \star}$ & Better FSR \\
\hline$\sigma_{\text {had }}^{0}[\mathrm{pb}]$ & $\sigma_{\text {had }}^{0}$ & $37\{25\}$ & $0.1[4.0]$ & $6 \times 3^{\star}$ & Better lumi MC \\
\hline$N_{v} \times 10^{3}$ & $\sigma\left(M_{Z}\right)$ & $8\{6\}$ & $0.005[1.0]$ & $6 \times 3^{\star \star}$ & CEEX in lumi MC \\
\hline$N_{v} \times 10^{3}$ & $Z \gamma$ & $150\{60\}$ & $0.8[<1]$ & $60 \times 3^{\star \star}$ & $\mathcal{O}\left(\alpha^{2}\right)$ for $Z \gamma$ \\
\hline $\sin ^{2} \theta_{W}^{\mathrm{eff}} \times 10^{5}$ & $A_{\mathrm{FB}}^{\text {lept. }}$ & $53\{28\}$ & $0.3[0.5]$ & $55 \times 3^{\star \star}$ & h.o. and EWPOs \\
\hline $\sin ^{2} \theta_{W}^{\mathrm{eff}} \times 10^{5}$ & $\left\langle\mathcal{P}_{\tau}\right\rangle, A_{\mathrm{FB}}^{\mathrm{pol}, \tau}$ & $41\{12\}$ & $0.6[<0.6]$ & $20 \times 3^{\star \star}$ & Better $\tau$ decay MC \\
\hline$M_{W}[\mathrm{MeV}]$ & Mass rec. & $33\{6\}$ & $0.5[0.3]$ & $12 \times 3^{\star \star \star}$ & QED at threshold \\
\hline$A_{\mathrm{FB}, \mu}^{M_{Z} \pm 3.5 \mathrm{GeV}} \times 10^{5}$ & $\frac{d \sigma}{d \cos \theta}$ & $2000\{100\}$ & $1.0[0.3]$ & $100 \times 3^{\star \star \star}$ & Improved IFI \\
\hline
\end{tabular}

in the $\mathrm{MC}$ programs certain provisions for fast evaluation of the changes of the SM predictions due to small variation of the SM input parameters, using the technique of weight differences.

It should be stressed that scenario proposed above is one of many possibilities. It will be the aim of the future studies and practice of the FCC-ee data analysis to decide about its final shape. For instance, in the above LEP-like scenario the main objective in the $(B) \rightarrow(C)$ transition is to encapsulate experimental data in the form of Born-like parametrization of the vector couplings of the $Z$ exchange, while background non-resonant exchange is parametrized in a rather simple way as photon exchange. One may consider departing from the above by means of including in the simplified spin amplitudes used in $(B) \rightarrow(C)$ stage most of the $\mathcal{O}\left(\alpha^{1}\right)$ EW corrections (like EW boxes), such that they are removed from the data. In this way EWPOs/EWPPs would represent more cleanly possible signals of new physics. One may also think about extending list of EWPPs with parameters residing in the nonresonant part of spin amplitudes. This would help to describe them in a refined way, again with the aim of exposing more efficiently possible signals of new physics in experimental data.

The above analysis only indicates certain possible avenues of reformulating the methodology of electroweak pseudoobservables and a more detailed analysis is badly needed. In the end, experimentalists in close collaboration with theorists will decide on how FCC-ee data will be analyzed.

\section{Summary}

The main results of our study are indicated in Table 2, where we have indicated for selected observables, the same as in
Table 1, the improvement factor needed in calculations of the QED effects in order to match the experimental precision anticipated in the FCC-ee experiment. We have also indicated explicitly the additional factor 3 necessary for these effects to become subdominant and the most important development to be done. We have also tried to rate how difficult it will be to achieve these targets. It is needless to say that many of the above estimates remain speculative and are not based on solid numerical results. However, this is always the case with estimates of the non-calculated higher order perturbative corrections, so they always have to be taken with a grain of salt. On the other hand, this kind of analysis is indispensable in planning directions and priorities of the future work.

Acknowledgements Authors would like to thank warmly J. Gluza, A. Freitas, P. Janot, W. Płaczek, Z. Wạs and B.F.L Ward for reading the manuscript critically.

Data Availability Statement This manuscript has no associated data or the data will not be deposited. [Authors' comment: All relevant data is included in the article body.]

Open Access This article is distributed under the terms of the Creative Commons Attribution 4.0 International License (http://creativecomm ons.org/licenses/by/4.0/), which permits unrestricted use, distribution, and reproduction in any medium, provided you give appropriate credit to the original author(s) and the source, provide a link to the Creative Commons license, and indicate if changes were made. Funded by SCOAP ${ }^{3}$.

\section{References}

1. M. Bicer et al., First look at the physics case of TLEP. JHEP 01(2014), 164 (2014). https://doi.org/10.1007/ JHEP01(2014)164. arXiv:1308.6176 
2. M. Mangano, et al., Future circular collider, vol. 1: physics opportunities. Submitted for publication to Eur. Phys. J. C. (2018), http://cds.cern.ch/record/2651294

3. M. Benedikt, et al., Future circular collider, vol. 2: the Lepton collider (FCC-ee). Submitted for publication to Eur. Phys. J. C (2019), http://cds.cern.ch/record/2651299

4. A. Blondel, et al., Standard Model Theory for the FCC-ee: the Tera-Z, in Mini Workshop on Precision EW and QCD Calculations for the FCC Studies: Methods and Techniques CERN, Geneva, Switzerland, January 12-13, 2018 (2018), arXiv:1809.01830

5. A. Blondel, A. Freitas, J. Gluza, S. Heinemeyer, S. Jadach, P. Janot, T. Riemann, Theory requirements and possibilities for the FCC-ee and other future high energy and precision frontier Lepton colliders (2019), arXiv:1901.02648

6. A. Blondel, J. Gluza, S. Jadach, P. Janot, T. Riemann (Eds.), Theory report on the 11th FCC-ee workshop, (2019). arXiv: 1905.05078

7. F.A. Berends, R. Kleiss, Distributions in the Process $e^{+} e^{-} \rightarrow$ $\mu^{+} \mu^{-}(\gamma)$. Nucl. Phys. B 177(1981), 237-262 (1981). https:// doi.org/10.1016/0550-3213(81)90390-4

8. F.A. Berends, W.L. van Neerven, G.J.H. Burgers, Higher order radiative corrections at LEP Energies. Nucl. Phys. B 297, 429 (1988) [Erratum: Nucl. Phys.B304,921(1988)]. https://doi.org/10.1016/0550-3213(88)90313-6. https://doi.org/ 10.1016/0550-3213(88)90662-1

9. S. Jadach, B.F.L. Ward, YFS2: the second order Monte Carlo for Fermion pair production at LEP/SLC with the initial state radiation of two hard and multiple soft photons. Comput. Phys. Commun. 56(1990), 351-384 (1990). https://doi.org/10.1016/ 0010-4655(90)90020-2

10. M. Skrzypek, Leading logarithmic calculations of QED corrections at LEP. Acta Phys. Polon. B 23(1992), 135-172 (1992)

11. S. Jadach, B.F.L. Ward, Z. Was, The precision Monte Carlo event generator KK for two fermion final states in $e^{+} e^{-}$collisions. Comput. Phys. Commun. 130(2000), 260-325 (2000). https://doi. org/10.1016/S0010-4655(00)00048-5. arXiv:hep-ph/9912214

12. D.Yu. Bardin, P. Christova, M. Jack, L. Kalinovskaya, A. Olchevski, S. Riemann, T. Riemann, ZFITTER v.6.21: a semianalytical program for fermion pair production in $e^{+} e^{-}$annihilation. Comput. Phys. Commun. 133(2001), 229-395 (2001). https://doi. org/10.1016/S0010-4655(00)00152-1. arXiv:hep-ph/9908433

13. G. Montagna, O. Nicrosini, F. Piccinini, G. Passarino, TOPAZ0 4.0: a new version of a computer program for evaluation of deconvoluted and realistic observables at LEP-1 and LEP-2. Comput. Phys. Commun. 117(1999), 278-289 (1999). https://doi.org/10. 1016/S0010-4655(98)00080-0. arXiv:hep-ph/9804211

14. S. Jadach, W. Placzek, M. Skrzypek, B.F.L. Ward, Z. Was, The Monte Carlo program KoralW version 15.1 and the concurrent Monte Carlo KoralW and YFSWW3 with all background graphs and first order corrections to W pair production. Comput. Phys. Commun. 140(2001), 475-512 (2001). https://doi.org/10.1016/ S0010-4655(01)00296-X. arXiv:hep-ph/0104049

15. A. Denner, S. Dittmaier, M. Roth, D. Wackeroth, RACOONWW1.3: a Monte Carlo program for four fermion production at $e^{+} e^{-}$colliders. Comput. Phys. Commun. 153(2003), 462-507 (2003). https://doi.org/10.1016/ S0010-4655(03)00205-4. arXiv:hep-ph/0209330

16. S. Jadach, B.F.L. Ward, Z. Was, Coherent exclusive exponentiation for precision Monte Carlo calculations. Phys. Rev. D 63(2001), 113009 (2001). https://doi.org/10.1103/PhysRevD.63. 113009. arXiv:hep-ph/0006359

17. D.R. Yennie, S.C. Frautschi, H. Suura, The infrared divergence phenomena and high-energy processes. Ann. Phys. 13(1961), 379-452 (1961). https://doi.org/10.1016/0003-4916(61)90151-8
18. S. Jadach, Yennie-Frautschi-Suura soft photons in Monte Carlo event generators, MPI-PAE/PTh 6/87, preprint of MPI Munchen, unpublished (1987)

19. S. Jadach, B.F.L. Ward, Exponentiation of Soft Photons in the Monte Carlo: the case of Bonneau and Martin. Phys. Rev. D 38, 2897 (1988). https://doi.org/10.1103/PhysRevD.39.1471.2. [Erratum: Phys. Rev.D39,1471(1989)]

20. M. Greco, G. Pancheri-Srivastava, Y. Srivastava, Radiative corrections for colliding beam resonances. Nucl. Phys. B 101(1975), 234-262 (1975). https://doi.org/10.1016/0550-3213(75)90304-1

21. S. Jadach, B.F.L. Ward, Z. Was, Coherent exclusive exponentiation CEEX: the case of the resonant $e^{+} e^{-}$collision. Phys. Lett. B 449(1999), 97-108 (1999). https://doi.org/10.1016/ S0370-2693(99)00038-6. arXiv:hep-ph/9905453

22. M. Greco, G. Pancheri-Srivastava, Y. Srivastava, Radiative corrections to $e^{+} e^{-} \rightarrow \mu^{+} \mu^{-}$Around the Z0. Nucl. Phys. B 171, 118 (1980). https://doi.org/10.1016/0550-3213(80)90363-6. [Erratum: Nucl. Phys. B197,543(1982)]

23. S. Jadach, B.F.L. Ward, Z. Was, The Monte Carlo program KORALZ, version 4.0, for the lepton or quark pair production at LEP/SLC energies. Comput. Phys. Commun. 79(1994), 503522 (1994). https://doi.org/10.1016/0010-4655(94)90190-2

24. S. Jadach, W. Placzek, E. Richter-Was, B.F.L. Ward, Z. Was, Upgrade of the Monte Carlo program BHLUMI for Bhabha scattering at low angles to version 4.04. Comput. Phys. Commun. 102(1997), 229-251 (1997). https://doi.org/10.1016/ S0010-4655(96)00156-7

25. S. Jadach, W. Płaczek, M. Skrzypek, B.F.L. Ward, Z. Wạs, The Monte Carlo event generator YFSWW3 version 1.16 for W pair production and decay at LEP2/LC energies. Comput. Phys. Commun. 140(2001), 432-474 (2001). https://doi.org/10.1016/ S0010-4655(01)00288-0. arXiv:hep-ph/0103163

26. S. Jadach, W. Placzek, M. Skrzypek, B.F.L. Ward, Z. Was, Monte Carlo program KoralW 1.42 for all four-fermion final states in $e^{+} e^{-}$collisions. Comput. Phys. Commun. 119(1999), 272311 (1999). https://doi.org/10.1016/S0010-4655(99)00219-2. arXiv:hep-ph/9906277

27. S. Jadach, W. Placzek, B.F.L. Ward, BHWIDE 1.00: O(alpha) YFS exponentiated Monte Carlo for Bhabha scattering at wide angles for LEP-1 / SLC and LEP-2. Phys. Lett. B 390, 298-308 (1997), also arXiv:hep-ph/9608412; the Monte Carlo program BHWIDE is available from http://cern.ch/ placzek (1997). arXiv:hep-ph/9608412. https://doi.org/10.1016/ S0370-2693(96)01382-2

28. S. Jadach, W. Placzek, B.F.L. Ward, Gauge invariant YFS exponentiation of (un)stable $\mathrm{Z}$ pair production at and beyond LEP-2 energies. Phys. Rev. D 56(1997), 6939-6941 (1997). https://doi. org/10.1103/PhysRevD.56.6939. arXiv:hep-ph/9705430

29. S. Schael et al., Precision electroweak measurements on the $Z$ resonance. Phys. Rep. 427(2006), 257-454 (2006). https://doi. org/10.1016/j.physrep.2005.12.006. arXiv:hep-ex/0509008

30. D. Yu. Bardin, M. Grunewald, G. Passarino, Precision calculation project report (1999), arXiv:hep-ph/9902452

31. S. Jadach, M. Skrzypek, B. Pietrzyk, On the precision of calculations of initial state radiation in the LEP Z line shape fits. Phys. Lett. B 456(1999), 77-79 (1999). https://doi.org/10.1016/ S0370-2693(99)00424-4

32. M. Greco, G. Pancheri-Srivastava, Y. Srivastava, Radiative effects for resonances with applications to colliding beam processes. Phys. Lett. B 56(1975), 367-372 (1975). https://doi.org/10.1016/ 0370-2693(75)90321-4

33. S. Jadach, S. Yost, QED Interference in charge asymmetry near the $\mathrm{Z}$ resonance at future electron-positron colliders (2018), arXiv:1801.08611 
34. D. Abbaneo, et al., A Combination of preliminary electroweak measurements and constraints on the standard model (2001), arXiv:hep-ex/0112021

35. G. Abbiendi et al., Photonic events with missing energy in $e^{+} e^{-}$collisions at $\mathrm{S}^{* *}(1 / 2)=189-\mathrm{GeV}$. Eur. Phys. J. C 18(2000), 253-272 (2000). https://doi.org/10.1007/ s100520000522. arXiv:hep-ex/0005002

36. S. Schael et al., Electroweak measurements in electron-positron collisions at W-Boson-pair energies at LEP. Phys. Rep. 532(2013), 119-244 (2013). https://doi.org/10.1016/j.physrep.2013.07.004. arXiv: 1302.3415

37. R. Tenchini, Electroweak precision measurements at the FCCee. Physics at FCC: overview of the Conceptual Design Report, CERN, March (2019), https://indico.cern.ch/event/789349/

38. S. Jadach, B. Pietrzyk, E. Tournefier, B.F.L. Ward, Z. Was, Initial final state interference in the $\mathrm{Z}$ line shape. Phys. Lett. B 465(1999), 254-259 (1999). https://doi.org/10.1016/ S0370-2693(99)01047-3. arXiv:hep-ph/9907547

39. S. Jadach, M. Skrzypek, M. Martinez, Light pair corrections to the Z line shape parameters. Phys. Lett. B 280(1992), 129-136 (1992). https://doi.org/10.1016/0370-2693(92)90786-4

40. A. Arbuzov, Higher order QED corrections to muon decay spectrum. JHEP 03(2003), 063 (2003). https://doi.org/10.1088/ 1126-6708/2003/03/063. arXiv:hep-ph/0206036

41. W. Beenakker, F.A. Berends, S.C. van der Marck, Large angle Bhabha scattering. Nucl. Phys. B 349(1991), 323-368 (1991). https://doi.org/10.1016/0550-3213(91)90328-U

42. E. Barberio, B. van Eijk, Z. Was, PHOTOS: a universal Monte Carlo for QED radiative corrections in decays. Comput. Phys. Commun. 66(1991), 115-128 (1991). https://doi.org/10.1016/ 0010-4655(91)90012-A

43. S. Jadach, W. Placzek, M. Skrzypek, B.F.L. Ward, Z. Was, Precision predictions for (un)stable $\mathrm{W}+\mathrm{W}-$ pair production at and beyond LEP-2 energies. Phys. Rev. D 65(2002), 093010 (2002). https://doi.org/10.1103/PhysRevD.65.093010. arXiv:hep-ph/0007012

44. M. Kobel, et al., Two-Fermion Production in Electron-Positron Collisions, in Proceedings, Monte Carlo Workshop: Report of the working groups on precision calculation for LEP-2 physics: CERN, Geneva, Switzerland, March 12-13, June 25-26, October 12-13 Oct 1999, (2000), arXiv:hep-ph/0007180. https://doi.org/ 10.5170/CERN-2000-009.269.http://weblib.cern.ch/abstract? CERN-2000-09-D

45. G. Bonneau, F. Martin, Hard photon emission in $e^{+} e^{-}$reactions. Nucl. Phys. B 27(1971), 381-397 (1971). https://doi.org/10.1016/ 0550-3213(71)90102-7

46. G. Altarelli, G. Martinelli, in Radiative Corrections to the ZO Line shape at LEP, ed. J. Ellis, R.D. Peccei Physics At Lep, vol. 1, (1986), pp. 47-57

47. G. Montagna, O. Nicrosini, F. Piccinini, The QED radiator at order $\alpha^{3}$. Phys. Lett. B 406(1997), 243-248 (1997). https://doi.org/10. 1016/S0370-2693(97)00705-3. arXiv:hep-ph/9611463

48. S. Jadach, M. Skrzypek, B.F.L. Ward, Is there a better way of exponentiating QED corrections? Phys. Lett. B 257(1991), 173178 (1991). https://doi.org/10.1016/0370-2693(91)90877-S

49. M. Przybycien, A Fifth order perturbative solution to the GribovLipatov equation. Acta Phys. Polon. B 24(1993), 1105-1114 (1993). arXiv:hep-th/9511029

50. J. Blümlein, A. De Freitas, C.G. Raab, K. Schönwald, The $O\left(\alpha^{2}\right)$ initial state QED corrections to $e^{+} e^{-}$annihilation to a neutral vector boson revisited. Phys. Lett. B 791(2019), 206-209 (2019). https://doi.org/10.1016/j.physletb.2019.02.038. arXiv: 1901.08018

51. J. Blumlein, A. De Freitas, W. van Neerven, Two-loop QED operator matrix elements with massive external fermion lines. Nucl.
Phys. B 855(2012), 508-569 (2012). https://doi.org/10.1016/j. nuclphysb.2011.10.009. arXiv:1107.4638

52. E.A. Kuraev, V.S. Fadin, On radiative corrections to $e^{+} e^{-}$single photon annihilation at high-energy. Sov. J. Nucl. Phys. 41, 466472 (1985). [Yad. Fiz.41,733(1985)] (1985)

53. S. Jadach, J.H. Kuhn, R.G. Stuart, Z. Wạs, QCD and QED corrections to the longitudinal polarization asymmetry. Z. Phys. C 38, 609 (1988). https://doi.org/10.1007/BF01624367. [Erratum: Z. Phys.C45,528(1990)]

54. B.F.L. Ward, S. Jadach, M. Melles, S.A. Yost, New results on the theoretical precision of the LEP/SLC luminosity. Phys. Lett. B 450(1999), 262-266 (1999). https://doi.org/10.1016/ S0370-2693(99)00104-5. arXiv:hep-ph/9811245

55. S. Jadach, W. Płaczek, M. Skrzypek, B.F.L. Ward, S .A. Yost, The path to $0.01 \%$ theoretical luminosity precision for the FCCee. Phys. Lett. B 790(2019), 314-321 (2019). https://doi.org/10. 1016/j.physletb.2019.01.012. arXiv:1812.01004

56. M. Dam, Lumical for fcc-ee and beam-background impactFCC Week (2018), https://indico.cern.ch/event/656491/

57. J. Alcaraz, et al., A Combination of preliminary electroweak measurements and constraints on the standard model (2006), arXiv:hep-ex/0612034

58. J. Alcaraz, et al., Precision electroweak measurements and constraints on the Standard Model (2007), arXiv:0712.0929

59. M. Böhm, et al., Forward-backward asymmetries, in LEP Physics Workshop Geneva, Switzerland, February 20, CERNTH-5536-89 (1989), pp. 203-234. https://doi.org/10.5170/ CERN-1989-008-V-1.203

60. S. Jadach, Z. Wass, Suppression of QED interference contributions to the charge asymmetry at the $Z^{0}$ resonance. Phys. Lett. B 219(1989), 103-106 (1989). https://doi.org/10.1016/ 0370-2693(89)90847-2

61. Z. Wạs, S. Jadach, First and higher order noninterference QED radiative corrections to the charge asymmetry at the $Z$ resonance. Phys. Rev. D 41(1990), 1425 (1990). https://doi.org/10. 1103/PhysRevD.41.1425

62. P.C. Christova, M. Jack, T. Riemann, Hard photon emission in $e^{+} e^{-} \rightarrow f \bar{f}$ with realistic cuts. Phys. Lett. B 456(1999), 264269 (1999). https://doi.org/10.1016/S0370-2693(99)00528-6. arXiv:hep-ph/9902408

63. P. Colas, R. Miquel, Z. Was, The neutrino anti-neutrino $\gamma$ cross-section and invisible width measurement at LEP. Phys. Lett. B 246(1990), 541-545 (1990). https://doi.org/10.1016/ 0370-2693(90)90646-N

64. G. Montagna, M. Moretti, O. Nicrosini, F. Piccinini, Single photon and multiphoton final states with missing energy at $e^{+} e^{-}$colliders. Nucl. Phys. B 541(1999), 31-49 (1999). https://doi.org/10. 1016/S0550-3213(98)00795-0. arXiv:hep-ph/9807465

65. M. Igarashi, N. Nakazawa, QED radiative corrections to the neutrino counting reaction $e^{+} e$. Nucl. Phys. B 288, 301-331 (1987). https://doi.org/10.1016/0550-3213(87)90217-3. [Erratum: Nucl. Phys.B294,1180(1987)]

66. A. Denner, S. Dittmaier, M. Roth, D. Wackeroth, Electroweak radiative corrections to e+ e- $\rightarrow \mathrm{W} \mathrm{W} \rightarrow 4$ fermions in double pole approximation: the RACOONWW approach. Nucl. Phys. B 587(2000), 67-117 (2000). https://doi.org/10.1016/ S0550-3213(00)00511-3. arXiv:hep-ph/0006307

67. E. Accomando, A. Ballestrero, E. Maina, WPHACT 2.0: a fully massive Monte Carlo generator for four fermion physics at $e^{+} e^{-}$colliders. Comput. Phys. Commun. 150(2003), 166196 (2003). https://doi.org/10.1016/S0010-4655(02)00685-9. arXiv:hep-ph/0204052

68. M.W. Grunewald, et al., Reports of the Working Groups on Precision Calculations for LEP2 Physics: Proceedings. Four fermion production in electron positron collisions (2000), arXiv:hep-ph/0005309 
69. M. Skrzypek, S. Jadach, M. Martinez, W. Placzek, Z. Was, Initial state QED corrections to $\mathrm{W}$ pair production at LEP2 / NLC: Monte Carlo versus semianalytical approach. Phys. Lett. B 372(1996), 289-298 (1996). https://doi.org/10.1016/ 0370-2693(96)00080-9

70. S. Jadach, W. Placzek, M. Skrzypek, B.F.L. Ward, Z. Was, On theoretical uncertainties of the $\mathrm{W}$ boson mass measurement at LEP-2. Phys. Lett. B 523(2001), 117-126 (2001). https://doi.org/ 10.1016/S0370-2693(01)01310-7. arXiv:hep-ph/0109072

71. A.P. Chapovsky, V.A. Khoze, Screened Coulomb ansatz for the nonfactorizable radiative corrections to the off-shell $\mathrm{W}+\mathrm{W}-$ production. Eur. Phys. J. C 9(1999), 449-457 (1999). https://doi.org/ 10.1007/s100529900070. arXiv:hep-ph/9902343

72. S. Jadach, W. Placzek, M. Skrzypek, B.F.L. Ward, Z. Was, Exact O (alpha) gauge invariant YFS exponentiated Monte Carlo for (un)stable $W^{+} W^{-}$production at and beyond LEP-2 energies. Phys. Lett. B 417(1998), 326-336 (1998). https://doi.org/10. 1016/S0370-2693(97)01253-7. arXiv:hep-ph/9705429

73. J. Fleischer, F. Jegerlehner, M. Zralek, Radiative corrections to helicity amplitudes for $\mathrm{W}$ pair production in $e^{+} e^{-}$annihilation. Z. Phys. C 42(1989), 409 (1989). https://doi.org/10.1007/ BF01548446

74. K. Kolodziej, M. Zralek, Helicity amplitudes for spin 0 or spin 1 boson production in $e^{+} e^{-}$annihilation. Phys. Rev. D 43(1991), 3619-3625 (1991). https://doi.org/10.1103/PhysRevD.43.3619

75. J. Fleischer, K. Kolodziej, F. Jegerlehner, W pair production in $e^{+} e^{-}$annihilation: radiative corrections including hard bremsstrahlung. Phys. Rev. D 47(1993), 830-836 (1993). https:// doi.org/10.1103/PhysRevD.47.830

76. J. Fleischer, F. Jegerlehner, K. Kolodziej, G.J. van Oldenborgh, EEWW: a generator for $e^{+} e^{-} \rightarrow W^{+} W^{-}$including one loop and leading photonic two loop corrections. Comput. Phys. Commun. 85(1995), 29-39 (1995). https://doi.org/10.1016/ 0010-4655(94)00113-G. arXiv:hep-ph/9405380

77. A. Denner, S. Dittmaier, M. Roth, L.H. Wieders, Complete electroweak $\mathrm{O}$ (alpha) corrections to charged-current $e^{+} e^{-} \rightarrow$ 4 fermion processes. Phys. Lett. B 612, 223-232 (2005). https://doi.org/10.1016/j.physletb.2005.03.007. [Erratum: Phys. Lett.B704,667(2011)], arXiv:hep-ph/0502063

78. A. Denner, S. Dittmaier, M. Roth, L.H. Wieders, Electroweak corrections to charged-current $e^{+} e^{-} \rightarrow 4$ fermion processes: technical details and further results. Nucl. Phys. B 724, 247-294 (2005). https://doi.org/10.1016/j.nuclphysb.2005.06.033. [Erratum: Nucl. Phys.B854,504(2012)] (2005), arXiv:hep-ph/0505042

79. A. Denner, S. Dittmaier, M. Roth, D. Wackeroth, Off-shell W pair production: universal versus nonuniversal corrections, in Proceedings, 5th International Symposium on Radiative CorrectionsRADCOR 2000 (2001). arXiv:hep-ph/0101257. http://www.slac. stanford.edu/econf/C000911/

80. W. Placzek, Precision predictions for $\mathrm{W}$ pair production at LEP-2. 172-176 (2002) [Nucl. Phys. Proc. Suppl.117,172(2003)]. arXiv:hep-ph/0208138. https://doi. org/10.1016/S0920-5632(03)90518-4

81. A. Denner, S. Dittmaier, M. Roth, D. Wackeroth, O(alpha) corrections to e+ e- $\rightarrow \mathrm{W} \mathrm{W} \rightarrow$ four fermions (+ gamma): first numerical results from RACOON W W. Phys. Lett. B 475(2000), 127134 (2000). https://doi.org/10.1016/S0370-2693(00)00059-9. arXiv:hep-ph/9912261

82. S. Catani, M.H. Seymour, A general algorithm for calculating jet cross-sections in NLO QCD. Nucl. Phys. B 485, 291-419 (1997). https://doi.org/10.1016/S0550-3213(96)00589-5. [Erratum: Nucl. Phys.B510,503(1998)] (1997), arXiv:hep-ph/9605323

83. S. Jadach, Z. Was, R. Decker, J.H. Kuhn, The tau decay library TAUOLA: version 2.4. Comput. Phys. Commun. 76(1993), 361380 (1993). https://doi.org/10.1016/0010-4655(93)90061-G
84. W. Placzek, S. Jadach, Multiphoton radiation in leptonic W boson decays. Eur. Phys. J. C 29(2003), 325-339 (2003). https://doi.org/ 10.1140/epjc/s2003-01223-4. arXiv:hep-ph/0302065

85. M. Beneke, P. Falgari, C. Schwinn, A. Signer, G. Zanderighi, Four-Fermion production near the $\mathrm{W}$ pair production threshold. Nucl. Phys. B 792(2008), 89-135 (2008). https://doi.org/10.1016/ j.nuclphysb.2007.09.030. arXiv:0707.0773

86. S. Actis, M. Beneke, P. Falgari, C. Schwinn, Dominant NNLO corrections to four-Fermion production near the W-pair production threshold. Nucl. Phys. B 807(2009), 1-32 (2009). https://doi. org/10.1016/j.nuclphysb.2008.08.006. arXiv:0807.0102

87. W. Beenakker, G.J. van Oldenborgh, A. Denner, S. Dittmaier, J. Hoogland, R. Kleiss, C.G. Papadopoulos, G. Passarino, The Fermion loop scheme for finite width effects in $e^{+} e^{-}$annihilation into four fermions. Nucl. Phys. B 500(1997), 255298 (1997). https://doi.org/10.1016/S0550-3213(97)00316-7. arXiv:hep-ph/9612260

88. A. Freitas, Higher-order electroweak corrections to the partial widths and branching ratios of the $\mathrm{Z}$ boson. JHEP 04(2014), 070 (2014). https://doi.org/10.1007/JHEP04(2014)070. arXiv: 1401.2447

89. A. Freitas, Electroweak precision tests in the LHC era and Zdecay form factors at two-loop level. in Proceedings, 12th DESY Workshop on Elementary Particle Physics: Loops and Legs in Quantum Field Theory (LL2014): Weimar, Germany, April 27May 2 (2014). arXiv:1406.6980

90. I. Dubovyk, A. Freitas, J. Gluza, T. Riemann, J. Usovitsch, Complete electroweak two-loop corrections to $\mathrm{Z}$ boson production and decay. Phys. Lett. B 783(2018), 86-94 (2018). https://doi.org/10. 1016/j.physletb.2018.06.037. arXiv:1804.10236

91. I. Dubovyk, A. Freitas, J. Gluza, T. Riemann, J. Usovitsch, Electroweak pseudo-observables and Z-boson form factors at two-loop accuracy (2019), arXiv:1906.08815

92. Z. Was, Tau event generators-for precision tests of SM at FCC, in 11th FCC-ee workshop: Theory and experiments (2019), https:// indico.cern.ch/event/766859/

93. M. Tanabashi et al., Review of particle physics. Phys. Rev. D 98(3), 030001 (2018). https://doi.org/10.1103/PhysRevD.98. 030001

94. S. Jadach, B.F.L. Ward, Semianalytical third order calculations of the small angle Bhabha cross-sections. Acta Phys. Polon. B 28(1997), 1907-1979 (1997)

95. G. Balossini, C.M. Carloni Calame, G. Montagna, O. Nicrosini, F. Piccinini, Matching perturbative and parton shower corrections to Bhabha process at flavour factories. Nucl. Phys. B 758(2006), 227-253 (2006). https://doi.org/10.1016/j.nuclphysb. 2006.09.022. arXiv:hep-ph/0607181

96. G. Abbiendi et al., Measuring the leading hadronic contribution to the muon g-2 via $\mu$ e scattering. Eur. Phys. J. C 77(3), 139 (2017). https://doi.org/10.1140/epjc/s10052-017-4633-z. arXiv: 1609.08987

97. S. Jadach, M. Melles, B.F.L. Ward, S.A. Yost, Exact results on O (alpha) corrections to the single hard bremsstrahlung process in low angle Bhabha scattering in the SLC / LEP energy regime. Phys. Lett. B 377(1996), 168-176 (1996). https://doi.org/ 10.1016/0370-2693(96)00354-1. arXiv:hep-ph/9603248

98. A.A. Penin, Two-loop photonic corrections to massive Bhabha scattering. Nucl. Phys. B 734(2006), 185-202 (2006). https://doi. org/10.1016/j.nuclphysb.2005.11.016. arXiv:hep-ph/0508127

99. A.A. Penin, Two-loop corrections to Bhabha scattering. Phys. Rev. Lett. 95(2005), 010408 (2005). https://doi.org/10.1103/ PhysRevLett.95.010408. arXiv:hep-ph/0501120

100. T. Becher, K. Melnikov, Two-loop QED corrections to Bhabha scattering. JHEP 06(2007), 084 (2007). https://doi.org/10.1088/ 1126-6708/2007/06/084. arXiv:0704.3582 
101. S. Jadach, M. Skrzypek, B.F.L. Ward, Soft pairs corrections to low angle Bhabha scattering: YFS Monte Carlo approach. Phys. Rev. D 55(1997), 1206-1215 (1997). https://doi.org/10.1103/ PhysRevD.55.1206

102. S. Jadach, M. Skrzypek, B.F.L. Ward, Soft pairs real and virtual infrared functions in QED. Phys. Rev. D 49(1994), 1178-1182 (1994). https://doi.org/10.1103/PhysRevD.49.1178

103. P. Janot, Direct measurement of $\alpha_{Q E D}\left(m_{Z}^{2}\right)$ at the FCC-ee. JHEP 02, 053 (2016). https://doi.org/10.1007/JHEP02(2016)053. [Erratum: JHEP11,164(2017)] (2016), arXiv:1512.05544

104. A. Blondel, S. Jadach, QED corrections to high precision measurement of the $\mathrm{Z}$ invisible width at electron-positron collider, IFJPAN-IV-2019-4 (in preparation)

105. S. Jadach, B.F.L. Ward, Z. Was, The Monte Carlo program KORALZ, version 3.8, for the lepton or quark pair production at LEP / SLC energies. Comput. Phys. Commun. 66(1991), 276292 (1991). https://doi.org/10.1016/0010-4655(91)90077-X

106. B. Marina, Update on W mass measurement studies at FCC-ee: FCC Week 2019, June 24-28 (2019), https://indico.cern.ch/event/ 727555/ (2019)
107. G. Altarelli, T. Sjostrand, F. Zwirner (Eds.), Physics at LEP2: vol. 1 (1996), http://www.slac.stanford.edu/spires/find/books/www? $\mathrm{cl}=\mathrm{QCD} 162: \mathrm{L} 2: 1995$

108. R. Tenchini, FCC-ee physics \& experiments CDR plan and status. FCC WEEK 2017, Berlin 29.05-2.06 (2017), https://indico.cern. $\mathrm{ch} /$ event/556692/

109. M. Czakon, The Muon decay at the two loop level of the electroweak interactions, eConf C030626 FRAP22 (2003), arXiv:hep-ph/0309315

110. S. Jadach, QED Exponentiation for Charged Instable Particles. Seminar at DESY Zeuthen, (2001), http://nz42.ifj.edu.pl/user/ jadach/main/talks/ (unpublished)

111. S. Jadach, W. Płaczek, M. Skrzypek, QED exponentiation for quasi-stable charged particles: the $e^{-} e^{+} \rightarrow W^{-} W^{+}$process (2019), arXiv:1906.09071 